Research Paper

\title{
Compound Kushen injection combined with platinum- based chemotherapy for stage III/IV non-small cell lung cancer: A meta-analysis of 37 RCTs following the PRISMA guidelines
}

\author{
Hongwei Chen ${ }^{1,2 \#}$, Xiaojun Yao ${ }^{1,3 \#, ~ T i n g ~ L i ~}{ }^{1,3}$, Christopher Wai-Kei Lam ${ }^{1,2}$, Ruonan Zhang ${ }^{1,3}$, Huixia

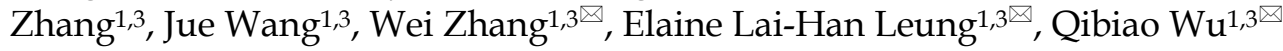 \\ 1. State Key Laboratory of Quality Research in Chinese Medicines, Macau University of Science and Technology, Avenida Wai Long, Taipa, Macau 999078, \\ China. \\ 2. Faculty of Medicine, Macau University of Science and Technology, Avenida Wai Long, Taipa, Macau 999078, China. \\ 3. Faculty of Chinese Medicine, Macau University of Science and Technology, Avenida Wai Long, Taipa, Macau 999078, China. \\ \# Equal contributors and co-first authors. \\ $\triangle$ Corresponding authors: Wei Zhang, E-mail: wzhang@must.edu.mo; Elaine Lai-Han Leung, E-mail: lhleung@must.edu.mo); Qibiao Wu, E-mail: \\ qbwu@must.edu.mo.
}

(c) The author(s). This is an open access article distributed under the terms of the Creative Commons Attribution License (https://creativecommons.org/licenses/by/4.0/). See http://ivyspring.com/terms for full terms and conditions.

Received: 2019.09.13; Accepted: 2019.12.09; Published: 2020.01.20

\begin{abstract}
Objective: Compound Kushen injection (CKI), one of the commonly used antitumor Chinese patent medicines, has been widely prescribed as adjunctive treatment to platinum-based chemotherapy (PBC) in patients with advanced non-small cell lung cancer (NSCLC). However, the efficacy and safety of this combination therapy for advanced NSCLC remain controversial. The objective of this study is to evaluate the effects of CKI combined with PBC on patients with stage III/IV non-small cell lung cancer.

Methods: A systematic review and meta-analysis were performed following the PRISMA (Preferred Reported Items for Systematic Review and Meta-analysis) guidelines. All randomized controlled trials (RCTs) comparing CKI in combination with PBC versus $\mathrm{PBC}$ alone were retrieved and assessed for inclusion. Analyses were performed using Review Manager 5.3 (Copenhagen: The Nordic Cochrane Centre, The Cochrane Collaboration, 2014), Comprehensive Meta-Analysis 3.0 (Biostat, Englewood, NJ, United States; 2016) and Trial Sequential Analysis software (TSA) (Copenhagen Trial Unit, Centre for Clinical Intervention Research, Copenhagen, Denmark; 2011). The disease control rate (DCR) was regarded as the primary outcome, and the objective response rate (ORR), quality of life (QOL), survival rate, and toxicities were the secondary outcomes.

Results: Thirty-seven trials, recruiting 3,272 patients with stage III/IV NSCLC, were included. The results showed that, CKI combined with PBC resulted in significant improvements in DCR $(R R=1.11,95 \% \mathrm{Cl}$ 1.07 to $1.15, \mathrm{P}<0.00001), \mathrm{ORR}(\mathrm{RR}=1.30,95 \% \mathrm{Cl} 1.20$ to $1.40, \mathrm{P}<0.00001), \mathrm{QOL}(\mathrm{RR}=1.73,95 \% \mathrm{Cl}$ 1.55 to $1.92, P<0.00001)$, 1-year survival rate $(R R=1.51,95 \% \mathrm{Cl} 1.18$ to $1.94, P=0.001)$, and a $58 \%$ decline in the incidence of severe toxicities ( $R R=0.42,95 \% \mathrm{Cl} 0.37$ to $0.49, \mathrm{P}<0.00001$ ).

Conclusions: From the available evidence, our data indicate that $\mathrm{CKI}$ plus platinum-based chemotherapy is more effective in improving clinical efficacy and alleviating the toxicity of chemotherapy than platinum-based chemotherapy alone in the treatment of stage III/IV NSCLC. However, considering the intrinsic limitations of the included trials, high-quality RCTs with survival outcomes are still needed to further confirm our findings.
\end{abstract}

Key words: Compound Kushen injection; platinum-based chemotherapy; non-small-cell lung carcinoma (NSCLC); systematic review; meta-analysis. 


\section{Introduction}

Worldwide, lung cancer remains the most common cancer and the leading cause of cancer-related mortality. In 2018, there were an estimated 2.1 million new lung cancer cases $(11.6 \%$ of the total cancer cases) and 1.8 million deaths (18.4\% of the total cancer deaths) [1-3]. The incidence and mortality rates of lung cancer have significantly increased in recent years, and lung cancer has become a major public health problem in the developing world, including China $[1,4,5]$.

Non-small cell lung cancer (NSCLC) accounts for approximately $85 \%$ of all lung cancer cases and about $66 \%$ of newly diagnosed NSCLC patients are already at stage III/IV [2]. Over the past decade, targeted therapy and immunotherapy have been dramatically changing the therapeutic scenario and providing better outcomes to advanced NSCLC patients identified based on their molecular profiles. Unfortunately, not all patients can benefit from these precision therapies, due to lack of an actionable biomarker (more than $40 \%$ of NSCLC patients) or to unavailability of genomic testing and/or precision therapies, especially for the many patients in the developing world. For those patients, platinum-based chemotherapy (PBC) is still the cornerstone of treatment and a commonly recommended choice [6-10]. Despite all efforts, the prognosis of stage IIIB/IV NSCLC remains extremely poor with a median survival of 7.9 months [11], and compared with precision therapies, the treatment with $\mathrm{PBC}$ alone is usually associated with worse survival, increased risk of toxic effects, and poor quality of life (QOL) $[7,9,12]$. Therefore, there is a pressing need to improve the efficacy and safety of $\mathrm{PBC}$ for those patients who are treated with $\mathrm{PBC}$ rather than precision therapies.

In China and some Asian countries, traditional Chinese medicines have been increasingly prescribed for advanced lung cancer patients in combination with PBC for synergistic interactions [13, 14]. Compound Kushen injection (CKI) is one of the commonly used anticancer Chinese patent medicines approved by the State Food and Drug Administration of China (Drug Approval Number: Z14021231) for the treatment of various cancers [15].

CKI is a mixture of natural compounds extracted from two medicinal herbs, Kushen (Radix Sophorae Flavescentis) and Baituling (Rhizoma Smilacis Glabrae). Active ingredients of CKI are matrine, oxymatrine, sophoridine, and N-methylcytisine [16, 17]. Many studies have shown that CKI and its active ingredients have notable anti-tumor activities $[17,18$, 19], such as inhibiting cancer cell proliferation, invasion and metastasis $[16,20,21]$, inducing tumor cell apoptosis [22, 23], reducing angiogenesis [21], inducing cell cycle arrest [20, 21, 23], inhibiting glycometabolism and amino acid metabolism [16], and reversing multidrug resistance [24, 25]. Besides, CKI can effectively increase immunologic function [26] and alleviate chemoradiotherapy-induced toxicity [21, 24, 25].

Emerging randomized controlled trials (RCTs) investigated the effects of CKI in combination with PBC on advanced NSCLC patients; however, the results were controversial. Some RCTs indicated that this combined therapy could improve the disease control rate (DCR), objective response rate (ORR), quality of life (QOL), and reduce adverse events [18, 19], but some trials found no significant changes in the above outcomes [27-32]. The effects of CKI combined with PBC for patients with stage III/IV NSCLC have never been systematically assessed. Therefore, it is necessary to assess the efficacy and safety of CKI combined with PBC for patients with stage III/IV NSCLC, aiming to provide optimal therapy for the specific subsets of patients.

\section{Materials and Methods}

We conducted this systematic review and meta-analysis following the PRISMA (Preferred Reported Items for Systematic Review and Meta-analysis) guidelines [33]. This study has been registered with the International Prospective Register of Systematic Reviews (PROSPERO): CRD42019134892. Ethical approval was not required because the research materials were published studies.

\section{Types of Studies}

All RCTs comparing CKI plus PBC versus PBC alone were selected and assessed for inclusion in this study.

\section{Inclusion criteria}

The participants included in this study should meet the following criteria: Diagnosis of stage III-IV NSCLC using the histopathological/cytological diagnostic criteria and TNM staging system [34-36] at least one bi-dimensionally measurable lesion; Karnofsky performance status (KPS) score [37] of at least 60; the range of Performance Status score from 0 to 2; life expectancy at least 3 months.

\section{Exclusion criteria}

The trials were not RCTs; sample size of either group was less than 30 patients; diagnosis was not NSCLC; the staging was not at stage III/IV; the NSCLC diagnosis was not specified accurately; the 
trials in which baseline data of the participants were inconsistent; there were no relevant outcome measures; the chemotherapy regimen was not platinum-based chemotherapy (PBC) or not clarified; radiotherapy, surgery, chemotherapy, immunotherapy, or Chinese medicine therapy other than CKI had been administered within three months before randomization; full-text articles or data was not available.

\section{Types of Interventions}

Experimental group: CKI plus PBC; control group: $\mathrm{PBC}$ only. In each $\mathrm{RCT}$, the $\mathrm{PBC}$ regimen used in both experimental and control groups was the same. All PBC regimens were eligible.

\section{Types of Outcome Measures}

The primary clinical endpoint was the disease control rate (DCR). Objective response rate (ORR), quality of life (QOL), and toxic effects were defined as the secondary outcomes. According to the WHO criteria for reporting results of cancer treatment [38, 39], ORR and DCR were used to assess the short-term effectiveness. Improvement of QOL was considered when KPS score increased by 10 points or more after treatment. Anti-tumor drug toxicity was evaluated and classified as grades 0 to 4 , according to Recommendations for Grading of Acute and Subacute Toxicity [38]. In this research, grades 3 and 4 toxicities were defined as severe toxicities.

Table 1. Principal characteristics of the studies included in the meta-analysis.

\begin{tabular}{|c|c|c|c|c|c|c|}
\hline \multirow[t]{2}{*}{ Reference } & \multirow[t]{2}{*}{ Design } & \multirow{2}{*}{$\begin{array}{l}\text { Sample } \\
\text { size } \\
(\mathrm{T} / \mathrm{C})\end{array}$} & \multirow[t]{2}{*}{ Outcomes measure } & \multicolumn{2}{|l|}{ Treatment group } & \multirow{2}{*}{$\begin{array}{l}\text { Control group } \\
\text { (Chemotherapy } \\
\text { regimen) }\end{array}$} \\
\hline & & & & Intervention & $\begin{array}{l}\text { Cycle number of } \\
\text { CKI plus PBC }\end{array}$ & \\
\hline Ai XY, 2016 [48] & $\mathrm{RCT}$ & $68 / 68$ & ORR, DCR, PFS, toxic effects & $\mathrm{CKI}+\mathrm{DP}$ & 2 & DP \\
\hline Chen H, 2012 [51] & $\mathrm{RCT}$ & $34 / 34$ & ORR, DCR, QOL, toxic effects & $\mathrm{CKI}+\mathrm{TP}$ & 3 & $\mathrm{TP}$ \\
\hline Chen H, 2009 [49] & $\mathrm{RCT}$ & $31 / 31$ & ORR, DCR & $\mathrm{CKI}+\mathrm{EP}$ & 2 & $\mathrm{EP}$ \\
\hline Chen LG, 2011 [50] & $\mathrm{RCT}$ & $40 / 40$ & ORR, DCR, QOL, toxic effects & $\mathrm{CKI}+\mathrm{DC}$ & 2 & $\mathrm{DC}$ \\
\hline Chen WF, 2015 [52] & $\mathrm{RCT}$ & $53 / 52$ & ORR, DCR, QOL, toxic effects & $\mathrm{CKI}+\mathrm{NP}$ & 2 & NP \\
\hline Dong J, 2012 [27] & $\mathrm{RCT}$ & $40 / 40$ & ORR, DCR & $\mathrm{CKI}+\mathrm{PP}$ & 4 & PP \\
\hline Duan P, 2009 [53] & $\mathrm{RCT}$ & $72 / 71$ & ORR, DCR, toxic effects & $\mathrm{CKI}+\mathrm{GP}$ & 3 & GP \\
\hline Hei X, 2016 [54] & $\mathrm{RCT}$ & $34 / 34$ & ORR, DCR, QOL, toxic effects & $\mathrm{CKI}+\mathrm{TP}$ & 3 & $\mathrm{TP}$ \\
\hline $\mathrm{Hu}$ AL, 2014 [55] & $\mathrm{RCT}$ & $46 / 46$ & ORR, DCR, toxic effects & $\mathrm{CKI}+\mathrm{TP}$ & 3 & $\mathrm{TP}$ \\
\hline Huang ZF, 2007 [56] & $\mathrm{RCT}$ & $30 / 30$ & ORR, DCR & $\mathrm{CKI}+\mathrm{NP}$ & 2 & NP \\
\hline Li CJ, 2011 [57] & $\mathrm{RCT}$ & $40 / 40$ & ORR, DCR & $\mathrm{CKI}+\mathrm{TP}$ & 2 & $\mathrm{TP}$ \\
\hline Liu GH, 2012 [60] & $\mathrm{RCT}$ & $60 / 60$ & ORR, DCR, QOL, toxic effects & CKI + GP & 2 & GP \\
\hline Liu YT, 2010 [59] & RCT & $32 / 32$ & ORR, DCR, QOL, toxic effects & $\mathrm{CKI}+\mathrm{TP}$ & 2 & $\mathrm{TP}$ \\
\hline Liu Y, 2009 [58] & $\mathrm{RCT}$ & $44 / 40$ & ORR, DCR, QOL & $\mathrm{CKI}+\mathrm{GP}$ & 2 & GP \\
\hline Long SP, 2008 [61] & $\mathrm{RCT}$ & $60 / 57$ & ORR, DCR, toxic effects & $\mathrm{CKI}+\mathrm{TP}$ & 2 & $\mathrm{TP}$ \\
\hline Lu WL, 2017 [18] & RCT & $60 / 60$ & ORR, DCR, QOL & $\mathrm{CKI}+\mathrm{GP}$ & 4 & GP \\
\hline Lu Y, 2005 [28] & $\mathrm{RCT}$ & $32 / 30$ & ORR, DCR, QOL & $\mathrm{CKI}+\mathrm{EP}$ & 3 & $\mathrm{EP}$ \\
\hline Pang DS, 2011 [29] & RCT & $32 / 30$ & ORR, DCR, QOL, toxic effects & $\mathrm{CKI}+\mathrm{TP}$ & 3 & $\mathrm{TP}$ \\
\hline Sang XW, 2012 [62] & RCT & $54 / 52$ & ORR, DCR, QOL, 1-year survival rate, toxic effects & $\mathrm{CKI}+\mathrm{NP}$ & 2 & NP \\
\hline Song Y, 2014 [19] & $\mathrm{RCT}$ & $30 / 30$ & ORR, DCR, QOL, toxic effects & $\mathrm{CKI}+\mathrm{GP}$ & 2 & GP \\
\hline Su WZ, 2007 [63] & $\mathrm{RCT}$ & $50 / 30$ & ORR, DCR, toxic effects & $\mathrm{CKI}+\mathrm{NP}$ & 2 & NP \\
\hline Wang ZX, 2009 [64] & $\mathrm{RCT}$ & $30 / 30$ & ORR, DCR, QOL, toxic effects & $\mathrm{CKI}+\mathrm{GP}$ & 2 & GP \\
\hline Wang H, 2009 [30] & $\mathrm{RCT}$ & $76 / 68$ & ORR, DCR, toxic effects & $\mathrm{CKI}+\mathrm{TC}$ & 2 & $\mathrm{TC}$ \\
\hline Wang HJ, 2012 [68] & $\mathrm{RCT}$ & $40 / 38$ & ORR, DCR & $\mathrm{CKI}+\mathrm{TP}$ & 2 & $\mathrm{TP}$ \\
\hline Wang LY, 2009 [65] & RCT & $45 / 45$ & ORR, DCR, QOL, toxic effects & $\mathrm{CKI}+\mathrm{NP}$ & 4 & $\mathrm{NP}$ \\
\hline Wang SF, 2016 [69] & $\mathrm{RCT}$ & $51 / 46$ & ORR, DCR, QOL & $\mathrm{CKI}+\mathrm{TP}$ & 2 & $\mathrm{TP}$ \\
\hline Wang YJ, 2015 [66] & RCT & $30 / 30$ & ORR, DCR, QOL, toxic effects & $\mathrm{CKI}+\mathrm{TP}$ & 4 & $\mathrm{TP}$ \\
\hline Wang YB, 2015 [67] & $\mathrm{RCT}$ & $56 / 52$ & ORR, DCR, QOL & $\mathrm{CKI}+\mathrm{TP}$ & 2 & $\mathrm{TP}$ \\
\hline Wu YJ, $2011[70]$ & $\mathrm{RCT}$ & $34 / 42$ & ORR, DCR, QOL & $\mathrm{CKI}+\mathrm{TP} / \mathrm{GP}$ & 2 & $\mathrm{TP} / \mathrm{GP}$ \\
\hline Wu HJ, 2006 [31] & RCT & $43 / 44$ & ORR, DCR, QOL, toxic effects & $\mathrm{CKI}+\mathrm{NP}$ & 3 & $\mathrm{NP}$ \\
\hline Xiao P, 2012 [32] & $\mathrm{RCT}$ & $53 / 50$ & ORR, DCR, QOL, toxic effects & $\mathrm{CKI}+\mathrm{GP}$ & 2 & GP \\
\hline Xu YQ, 2007 [73] & RCT & $36 / 30$ & ORR, DCR, QOL & $\mathrm{CKI}+\mathrm{TP}$ & 2 & $\mathrm{TP}$ \\
\hline Yu ZG, 2012 [74] & $\mathrm{RCT}$ & $50 / 40$ & ORR, DCR, QOL, toxic effects & $\mathrm{CKI}+\mathrm{GC}$ & 2 & GC \\
\hline Zhang JW, 2015 [71] & $\mathrm{RCT}$ & $60 / 60$ & ORR, DCR, QOL & $\mathrm{CKI}+\mathrm{GP}$ & 2 & GP \\
\hline Zhang JJ, 2015 [72] & $\mathrm{RCT}$ & $42 / 42$ & ORR, DCR, 1-year survival rate, 2-year survival rate & $\mathrm{CKI}+\mathrm{TP}$ & 3 & $\mathrm{TP}$ \\
\hline Zhao K, 2012 [75] & $\mathrm{RCT}$ & $43 / 43$ & ORR, DCR & $\mathrm{CKI}+\mathrm{GP}$ & 2 & GP \\
\hline Zhou X, 2010 [76] & RCT & $39 / 35$ & ORR, DCR, QOL, 1-year survival rate, toxic effects & CKI+ GP & 2 & GP \\
\hline
\end{tabular}




\section{Information Sources}

A comprehensive literature search was conducted by two independent researchers (HW Chen and HX Zhang). Published studies were retrieved in common databases including PubMed, Web of Science, ClinicalTrials.gov, Cochrane Library, EMBASE, China National Knowledge Infrastructure (CNKI), Wanfang Databases, the Chinese Scientific Journal Database, the Chinese Science Citation Database, and the Chinese Biomedical Literature Database. The last search date was April 20, 2019. In addition, we searched and evaluated the relevant systematic reviews and meta-analyses to select the potential studies from their references.

\section{Search Strategy}

The search details were conducted as follows (English database): \{("Carcinoma, Non-Small-Cell Lung" [Mesh] OR "Carcinoma, Non-Small-Cell Lung" [Title/Abstract] OR "Carcinomas, Non-Small-Cell Lung" [Title/Abstract] OR "Lung Carcinoma, Non-Small-Cell" [Title/Abstract] OR "Lung Carcinomas, Non-Small-Cell" [Title/Abstract] OR "Non-Small-Cell Lung Carcinomas" [Title/Abstract] OR "Non-small Cell Lung Cancer" [Title/Abstract] OR "Non-Small-Cell Lung Carcinoma" [Title/Abstract] OR "Non-Small Cell Lung Carcinoma" [Title/Abstract] OR "Carcinoma, Non-Small Cell Lung" [Title/Abstract] OR "Non-Small Cell Lung Cancer" [Title/Abstract]) AND ("Compound Kushen injection" [Title/Abstract] OR "Fufang Kushen injection" [Title/Abstract] OR "Yanshu injection")\}.Chinese databases (CNKI, etc.) searches: \{"feixiaoxibaofeiai" ("carcinoma, non-smallcell lung") AND ("Fufangkushenzhusheye" ("Compound Kushen injection" OR "Fufang Kushen injection" OR "Yanshu injection") AND Hualiao ("chemotherapy")\}.

\section{Study Selection}

Two independent reviewers (HW Chen and T Li) screened all the candidate articles on the basis of title and abstract. The full texts were retrieved for further assessment according to the inclusion and exclusion criteria. All inclusion disagreements were resolved by consensus.

\section{Data extraction}

Three reviewers (HW Chen, XJ Yao, and HX Zhang) independently rated the included RCTs and extracted the data. If a trial reported ambiguous or incomplete data, reviewers contacted the corresponding author via email and/or phone for further information. The intention-to-treat (ITT) analysis was used to analyze the results whenever available. The characteristics of all included RCTs are summarized in Table 1.

\section{Risk of Bias in Individual Trials}

Two independent reviewers (HW Chen and $\mathrm{T} \mathrm{Li}$ ) appraised the risk of bias in the included trials using the Cochrane Risk of Bias Tool for Randomized Controlled Trials [38]. The following criteria were used to evaluate bias in each trial: random sequence generation; concealment of allocation; blinding of participants and personnel; blinding of outcome assessment; incomplete data; selective reporting; and other bias. The risk of bias was classified as 'unclear', 'low' or 'high'.

\section{Summary Measures and Data Synthesis}

All analyses were performed using the Review Manager (RM) 5.3 (Copenhagen: The Nordic Cochrane Centre, The Cochrane Collaboration, 2014), the Comprehensive Meta-Analysis (CMA) 3.0 (Biostat, Englewood, NJ, United States; 2016) and the Trial Sequential Analysis (TSA) software (Copenhagen Trial Unit, Centre for Clinical Intervention Research, Copenhagen, Denmark; 2011). Dichotomous data were shown as the risk ratio (RR), risk difference (RD) or odds ratio (OR), and continuous data were shown as the weighted mean difference (WMD) or standardized mean difference (SMD) with a 95\% confidence intervals (CI). Heterogeneity was assessed using the $I^{2}$ statistic and $\mathrm{Chi}^{2}$ test. Substantial heterogeneity was considered when $I^{2}>50 \%$ or $P<0.01$. If the hypothesis of homogeneity was not rejected, a fixed-effects model was used to estimate the summary RR (OR or RD), WMD (or SMD) and 95\% CI; otherwise, a random-effects model was used [40-43].

\section{Risk of Bias across trials}

When the number of the included trials was $\geq 10$, Egger's test and the funnel plots were used to examine the potential bias in the RCTs included in the meta-analysis [40, 44-46].

\section{Additional analyses}

Sensitivity analysis, subgroup analysis and the Trial Sequential Analysis (TSA) were used to determine the robustness of results and calculate the required information size (RIS) in the meta-analysis [47]. A meta-regression analysis was also carried out to examine the potential heterogeneity and whether the moderator variables have an impact on the study effect size. 


\section{Results}

\section{Study Selection}

As shown in Figure 1, there were 2,035 records identified through the database search, 501 of them were duplicated and excluded. A total of 1,384 articles of case reports, reviews, letters, and basic researches were excluded after screening the title and the abstract. The full texts of 150 candidate papers were then screened and evaluated, and 113 were removed for the following reasons: unrelated with this research topic $(n=42)$; conference article $(n=1)$; not meeting inclusion criteria or meeting exclusion criteria $(\mathrm{n}=$ 70). Finally, 37 trials met the inclusion criteria. All the papers were in the Chinese language.

\section{Study Characteristics}

Thirty-seven RCTs recruiting 3,272 patients were included (Table 1) [18, 19, 27-32, 48-76]. All trials were conducted in China, and the articles were published from 2005 to 2017. All participants enrolled were patients with NSCLC at TMN stage III/IV. There were 1,670 and 1,602 patients in the experimental and control groups, respectively. The number of participants in each RCT varied from 60 to 144 . The ages of the participants ranged from 18 to 80 .

All RCTs included compared CKI combined with PBC versus PBC alone. Thirteen RCTs used TP (paclitaxel plus cisplatin), 10 RCTs used GP (gemcitabine plus cisplatin), 6 RCTs used NP (vinorelbine plus cisplatin), 2 RCTs used EP (Etoposide plus cisplatin), 1 RCT used DC (docetaxel plus carboplatin), 1 RCT used DP (docetaxel plus cisplatin), 1 RCT used TC (paclitaxel plus carboplatin), 1 RCT used PP (pemetrexed plus cisplatin) and 1 used RCT GC (Gemcitabine plus carboplatin), and 1 RCT used TP or GP regimens. In all experimental groups, CKI was synchronously administered with PBC for the patients. Twenty-five trials used 2 cycles of CKI for the patients in the experimental group, 8 trials used 3 cycles, and 4 trials used 4 cycles. The follow-up duration of the included RCTs varied from 1 to 30 months.
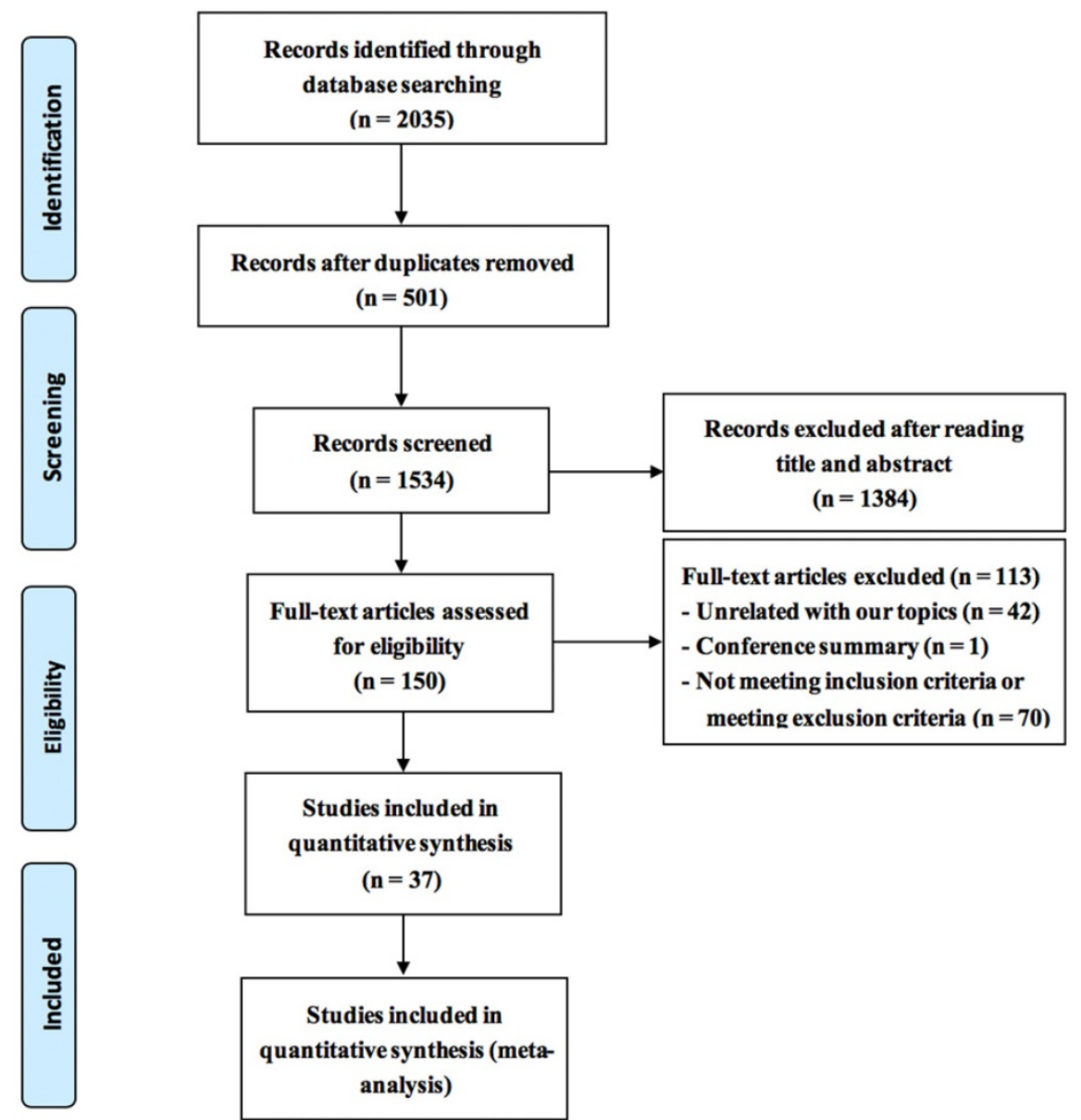

Figure 1. Preferred reporting items for systematic reviews and meta-analysis (PRISMA) search diagram. RCT = randomized controlled trial. 
Table 2. The methodologic quality of the included trials assessed using the Cochrane Risk of Bias Tool.

\begin{tabular}{|c|c|c|c|c|c|c|c|}
\hline Reference & $\begin{array}{l}\text { Random } \\
\text { sequence } \\
\text { generation }\end{array}$ & $\begin{array}{l}\text { Allocation } \\
\text { concealment }\end{array}$ & $\begin{array}{l}\text { Blinding of } \\
\text { participants and } \\
\text { personnel }\end{array}$ & $\begin{array}{l}\text { Blinding of } \\
\text { outcome } \\
\text { assessment }\end{array}$ & $\begin{array}{l}\text { Incomplete outcome } \\
\text { data }\end{array}$ & $\begin{array}{l}\text { Selective } \\
\text { reporting }\end{array}$ & Other bias \\
\hline Ai XY, 2016 [48] & $?$ & $?$ & $?$ & $?$ & + & + & $?$ \\
\hline Chen H, 2009 [49] & $?$ & $?$ & $?$ & $?$ & + & + & $?$ \\
\hline Chen LG, 2011 [50] & + & + & ? & $?$ & + & + & $?$ \\
\hline Chen H, 2012 [51] & + & + & $?$ & $?$ & + & + & $?$ \\
\hline Chen WF, 2015 [52] & + & + & ? & $?$ & + & + & $?$ \\
\hline Dong J, 2012 [27] & + & + & $?$ & $?$ & + & + & $?$ \\
\hline Duan P, 2009 [53] & $?$ & $?$ & ? & $?$ & + & + & $?$ \\
\hline Hei X, 2016 [54] & + & + & $?$ & $?$ & + & + & $?$ \\
\hline $\mathrm{Hu}$ AL, 2014 [55] & + & + & $?$ & ? & + & + & $?$ \\
\hline Huang ZF, 2007 [56] & + & + & $?$ & $?$ & + & + & $?$ \\
\hline Li CJ, 2011 [57] & + & + & ? & $?$ & + & + & $?$ \\
\hline Liu Y, 2009 [58] & $?$ & $?$ & $?$ & $?$ & + & + & $?$ \\
\hline Liu YT, 2010 [59] & + & + & ? & $?$ & + & + & $?$ \\
\hline Liu GH, 2012 [60] & + & + & $?$ & $?$ & + & + & $?$ \\
\hline Long SP, 2008 [61] & + & + & $?$ & ? & + & + & $?$ \\
\hline Lu Y, 2005 [28] & + & + & $?$ & $?$ & + & + & $?$ \\
\hline Lu WL, 2017 [18] & + & + & $?$ & $?$ & + & + & $?$ \\
\hline Pang DS, 2011 [29] & + & + & $?$ & $?$ & + & + & $?$ \\
\hline Sang XW, 2012 [62] & + & + & $?$ & ? & + & + & $?$ \\
\hline Song Y, 2014 [19] & + & + & $?$ & $?$ & + & + & $?$ \\
\hline Su WZ, 2007 [63] & $?$ & $?$ & $?$ & $?$ & + & + & $?$ \\
\hline Wang LY, 2009 [65] & + & + & $?$ & $?$ & + & + & $?$ \\
\hline Wang H, 2009 [30] & $?$ & $?$ & $?$ & $?$ & + & + & $?$ \\
\hline Wang ZX, 2009 [64] & + & + & $?$ & $?$ & + & + & $?$ \\
\hline Wang HJ, 2012 [68] & + & + & $?$ & $?$ & + & + & $?$ \\
\hline Wang SF, 2016 [69] & + & + & $?$ & $?$ & + & + & $?$ \\
\hline Wang YJ, 2015 [66] & + & + & $?$ & $?$ & + & + & $?$ \\
\hline Wang YB, 2015 [67] & + & + & $?$ & $?$ & + & + & $?$ \\
\hline Wu HJ, 2006 [31] & + & + & $?$ & $?$ & + & + & $?$ \\
\hline Wu YJ, 2011 [70] & + & + & $?$ & $?$ & + & + & $?$ \\
\hline Xiao P, 2012 [32] & $?$ & $?$ & $?$ & $?$ & + & + & $?$ \\
\hline Xu YQ, 2007 [73] & + & + & $?$ & $?$ & + & + & $?$ \\
\hline Yu ZG, 2012 [74] & $?$ & $?$ & $?$ & $?$ & + & + & $?$ \\
\hline Zhang JJ, 2015 [72] & + & + & $?$ & $?$ & + & + & $?$ \\
\hline Zhang JW, 2015 [71] & + & + & $?$ & $?$ & + & + & $?$ \\
\hline Zhao K, 2012 [75] & + & + & $?$ & $?$ & + & + & $?$ \\
\hline Zhou X, 2010 [76] & $?$ & $?$ & $?$ & $?$ & + & + & $?$ \\
\hline
\end{tabular}

$+=$ low risk of bias; $?=$ unclear risk of bias; $-=$ high risk of bias.

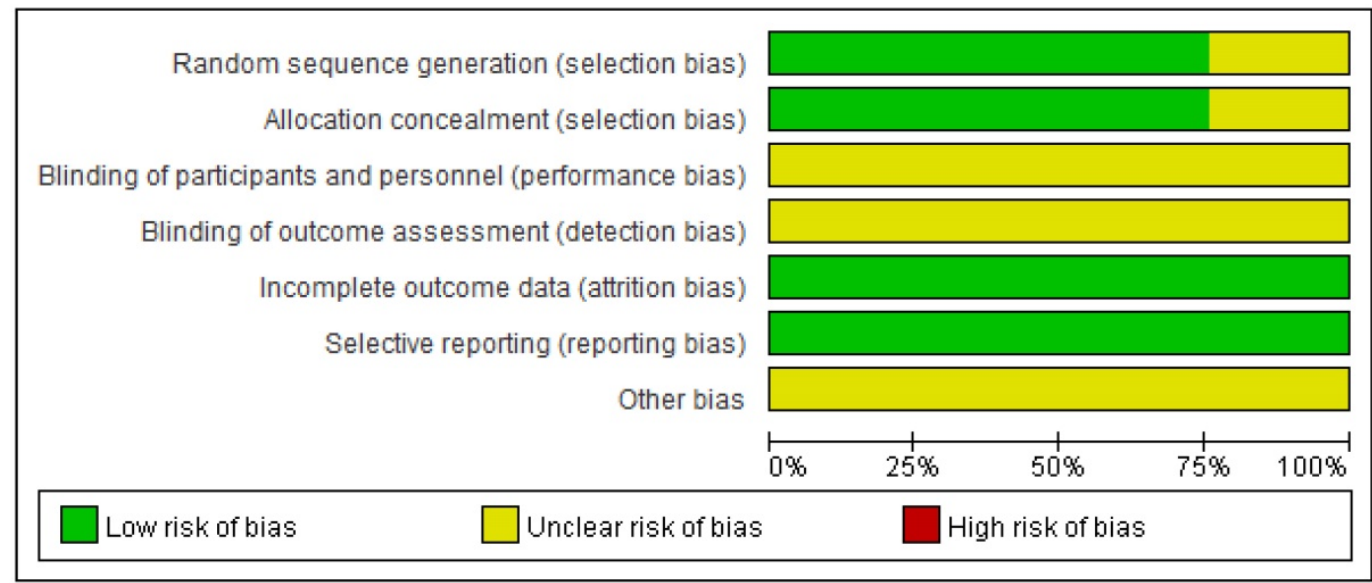

Figure 2. Risk of bias graph. 


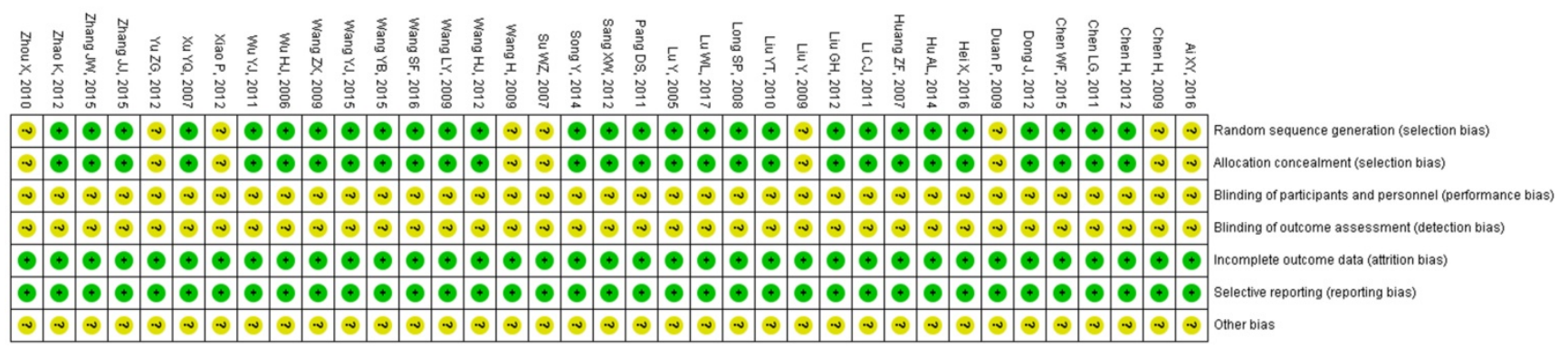

Figure 3. Risk of bias summary.

\section{Methodological quality}

The risk of bias of all included RCTs was evaluated and summarized in Table 2, Figures 2 and 3 . Randomization was used in all included studies, 28 studies clearly described appropriate randomization methods, risk of selection bias (random sequence generation) was low in these studies $[18,19,27-29,31$, 50-52, 54-57, 59-62, 64-73, 75]. Another 9 RCTs did not describe how the randomization was accomplished and the risk of selection bias was unclear $[30,32,48$, $49,53,58,63,74,76]$. In all included RCTs, blinding of participants/personnel/outcome assessment was unclear. The data of all included trials were complete, the withdrawals and/or dropouts of patients were described and the reasons were reported. The risk of reporting bias was low. Any other bias was not clear.

\section{Outcome measures}

The findings of the meta-analyses are summarized in Table 3.

\section{Disease control rate (DCR) and objective response rate (ORR)}

All 37 included RCTs reported changes in DCR and ORR after the interventions. The pooled data showed that, compared with PBC alone, CKI plus PBC significantly improved DCR ( RR $=1.11,95 \%$ CI 1.07 to $1.15, P<0.00001$; Figure 4$)$ and $\mathrm{ORR}(\mathrm{RR}=1.30$, 95\% CI 1.20 to 1.40, $P<0.00001$; Figure 5). There was statistical homogeneity for both outcomes (both $I^{2}=$ $0 \%$, and the fixed-effects model was used to combine the trials.

\section{Improvement of QOL}

Twenty-three trials investigated the effects of different interventions on the QOL $[18,19,28,29,31$, 32, 20-52, 53, 58-60, 64-67, 69, 70, 72-74, 76]. Pooling data from these 23 studies showed that, compared with PBC alone, CKI plus PBC significantly improved QOL $(\mathrm{RR}=1.73,95 \%$ CI 1.55 to $1.92, P<0.00001$; Figure 6). There was statistical homogeneity for this outcome $\left(I^{2}=0 \%\right)$, and the fixed-effects model was used to combine the trials.

\section{Survival rates and other survival outcomes}

Three trials reported the effect of CKI plus PBC on 1-year survival rate $[62,72,76]$ and the result of meta-analysis showed that, compared with PBC alone, CKI plus PBC significantly increased 1-year survival rate $(R R=1.51,95 \% \mathrm{CI} 1.18$ to $1.94, P=0.001$; Figure 7). There was statistical homogeneity for this outcome $\left(I^{2}=0 \%\right)$, and the fixed-effects model was used to combine the trials.

Only one trial reported that there was no significant difference in the 2-year survival rate between groups $(33.33 \%$ vs $23.81 \%, P>0.05)$ [72].

Several RCTs reported four other survival outcomes including overall survival (OS) [32], progression-free survival (PFS) [31, 32], median time to progression (mTTP) $[29,62,63]$, median survival time (MST) [29, 31, 62, 72], but, because of the unextractable data and/or the diversity of survival outcomes in the included RCTs, meta-analysis was not possible for these outcomes.

\section{Toxicities}

Twenty-four RCTs reported the effect of CKI on severe (grade 3 and 4) toxic effects according to the WHO criteria [38]. Compared with PBC alone, CKI plus $\mathrm{PBC}$ significantly reduced severe toxicities by $58 \%(\mathrm{RR}=0.42,95 \% \mathrm{CI} 0.37$ to $0.49, P<0.00001$; Figure 8).

The findings of the meta-analysis demonstrated that CKI plus PBC was associated with significant reductions in severe leukopenia $(\mathrm{RR}=0.44,95 \% \mathrm{CI}$ 0.35 to $0.55, P<0.00001)$, anemia ( $R R=0.22,95 \% \mathrm{CI}$ 0.12 to $0.38, P<0.00001)$, thrombocytopenia $(\mathrm{OR}=$ $0.51,95 \%$ CI 0.32 to $0.82, P=0.005)$, nausea and vomiting $(\mathrm{RR}=0.41,95 \% \mathrm{CI} 0.30$ to $0.56, P<0.00001)$, diarrhea (RR $=0.42,95 \% \mathrm{CI} 0.23$ to $0.77, P=0.004)$, stomatitis (RR $=0.31,95 \% \mathrm{CI} 0.13$ to $0.74, P=0.008)$, hair loss (RR $=0.47,95 \% \mathrm{CI} 0.24$ to $0.89, P=0.02)$. The differences between groups were statistically significant (Figure 8). 
However, for severe liver injury $(\mathrm{RR}=0.72,95 \%$ CI 0.43 to $1.20, P=0.21)$ and renal injury $(R R=0.41$, 95\% CI 0.11 to $1.58, P=0.20$ ), the differences between groups were not statistically significant (Figure 8).
There was no substantial heterogeneity for the above outcomes, and the fixed-effects model was used to combine the trials (Figure 8).

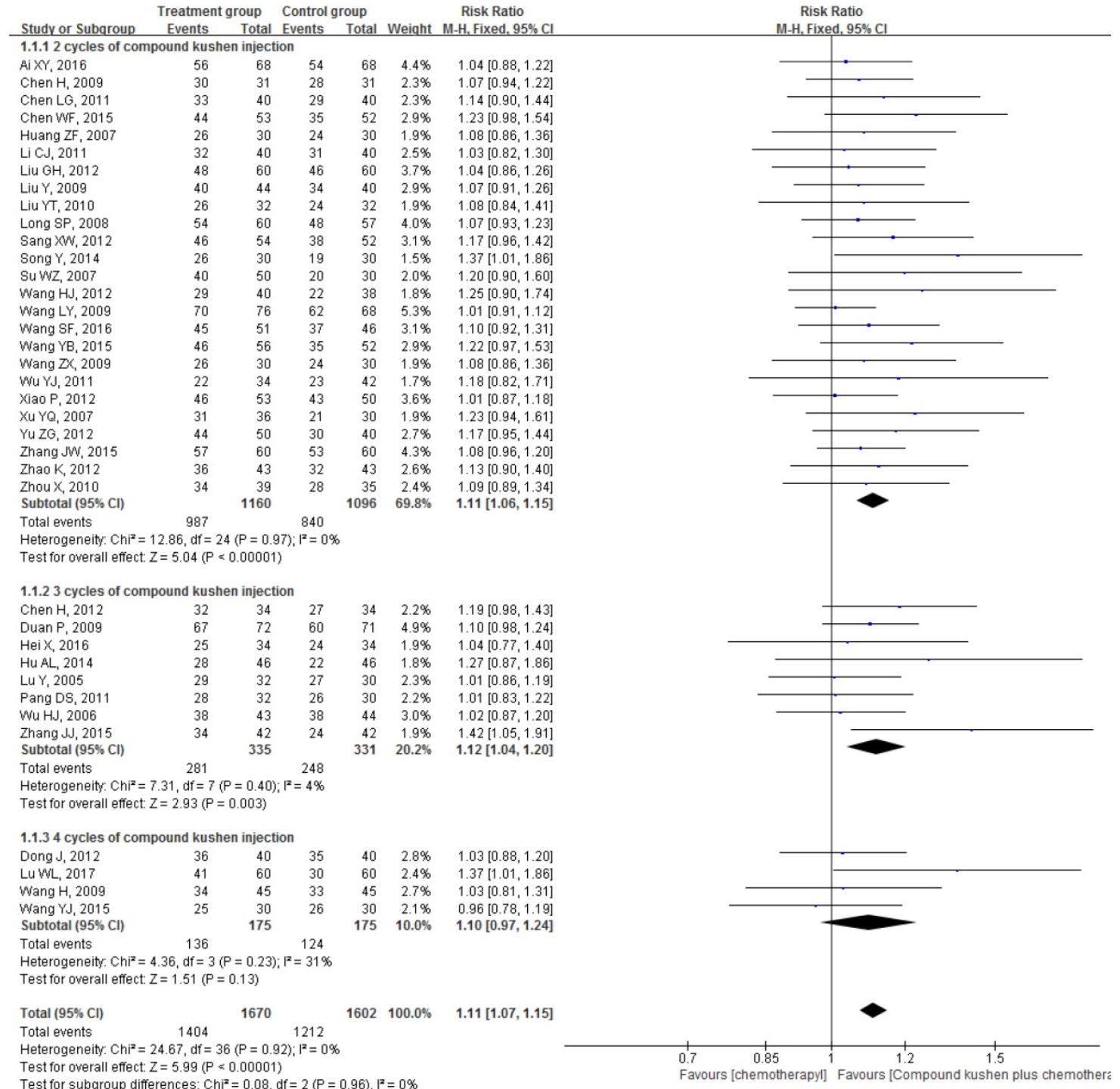

Figure 4. Forest plots showing a significant improvement in the DCR in the experimental group compared with that of the control group. Cl: Confidence Interval; CKI: Compound Kushen injection, PBC: platinum-based chemotherapy.

Table 3. Summary of the meta-analysis (pooled data across categories in the control group).

\begin{tabular}{|c|c|c|c|c|c|}
\hline Outcome or Subgroup & Studies & Participants & Statistical Method & Effect Estimate & $P$ \\
\hline \multirow[t]{3}{*}{$\overline{\mathrm{DCR}}$} & 37 & 3272 & Risk Ratio (M-H, Fixed, 95\% CI) & $1.11[1.07,1.15]$ & $<0.00001^{*}$ \\
\hline & & & Odds Ratio (M-H, Fixed, 95\% CI) & $1.73[1.45,2.07]$ & $<0.00001^{*}$ \\
\hline & & & Risk Difference (M-H, Fixed, 95\% CI) & $0.08[0.06,0.11]$ & $<0.00001^{*}$ \\
\hline \multirow[t]{3}{*}{ ORR } & 37 & 3272 & Risk Ratio (M-H, Fixed, 95\% CI) & $1.30[1.20,1.40]$ & $<0.00001^{*}$ \\
\hline & & & Odds Ratio (M-H, Fixed, 95\% CI) & $1.62[1.41,1.87]$ & $<0.00001^{*}$ \\
\hline & & & Risk Difference (M-H, Fixed, 95\% CI) & $0.11[0.08,0.15]$ & $<0.00001^{*}$ \\
\hline \multirow[t]{3}{*}{ QOL } & 23 & 1924 & Risk Ratio (M-H, Fixed, 95\% CI) & $1.73[1.55,1.92]$ & $<0.00001^{*}$ \\
\hline & & & Odds Ratio (M-H, Fixed, 95\% CI) & $2.78[2.29,3.37]$ & $<0.00001^{*}$ \\
\hline & & & Risk Difference (M-H, Fixed, 95\% CI) & $0.23[0.19,0.27]$ & $<0.00001^{*}$ \\
\hline \multirow[t]{3}{*}{ 1-year survival rate } & 3 & 134 & Risk Ratio (M-H, Fixed, 95\% CI) & $1.51[1.18,1.94]$ & $0.001^{*}$ \\
\hline & & & Odds Ratio (M-H, Fixed, 95\% CI) & $2.35[1.42,3.88]$ & $0.0008^{*}$ \\
\hline & & & Risk Difference (M-H, Fixed, 95\% CI) & $0.21[0.09,0.32]$ & $0.0005^{*}$ \\
\hline \multirow[t]{3}{*}{ Grade 3-4 toxicity } & 24 & 6842 & Risk Ratio (M-H, Fixed, 95\% CI) & $0.42[0.37,0.49]$ & $<0.00001^{*}$ \\
\hline & & & Odds Ratio (M-H, Fixed, 95\% CI) & $0.35[0.30,0.41]$ & $<0.00001^{*}$ \\
\hline & & & Risk Difference (M-H, Fixed, 95\% CI) & $-0.09[-0.11,-0.08]$ & $<0.00001^{*}$ \\
\hline
\end{tabular}

DCR: Disease control rate, ORR: Objective Response Rate, QOL: Quality of life, CI: Confidence Interval. 


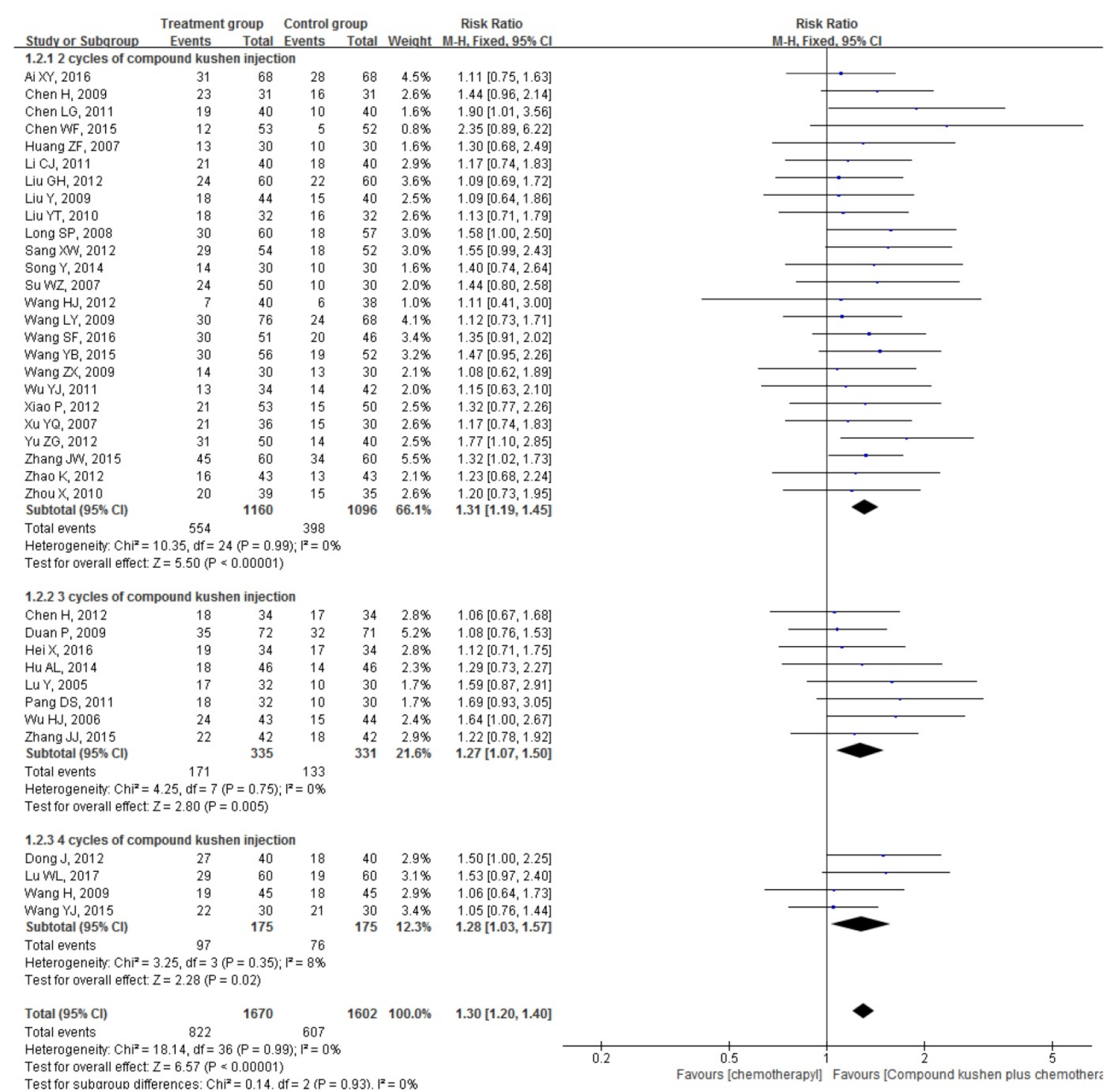

Figure 5. Forest plots showing a significant improvement in the ORR in the experimental group compared with that of the control group. Cl: Confidence Interval; CKI: Compound Kushen injection, PBC: platinum-based chemotherapy.

\section{Publication Bias}

The funnel plots of the DCR suggested possible publication bias in small trials (Figure 9A), the Egger's test $(P=0.745)$ of DCR demonstrated that there was no obvious publication bias. The funnel plots of ORR (Figure 9B), QOL (Figure 9C), and severe toxicities (Figure 9D) also showed possible publication bias due to small-study effects.

\section{Subgroup and sensitivity analyses}

With regard to DCR, the primary outcome, the pooled data showed that CKI plus PBC increased DCR significantly $(\mathrm{RR}=1.11,95 \% \mathrm{CI} 1.07$ to $1.15, P<$ 0.00001). Similar increases were observed when the subgroup and sensitivity analyses were performed based on the results of Cochrane Risk of Bias Tool (only including 28 RCTs with low risk of selection bias) $(\mathrm{RR}=1.12,95 \% \mathrm{CI} 1.08$ to $1.17, P<0.00001)[18$, 19, 27-29, 31，50-52, 54-57, 59-62, 64-73, 75], participants number ( $\geq 50$ in each group) $(R R=1.11$, 95\% CI 1.06 to $1.16, P<0.0001)$, the cycle number of CKI $[2$ cycles $(\mathrm{RR}=1.11,95 \%$ CI 1.06 to $1.15, P<$ $0.00001), 3$ cycles (RR $=1.12,95 \%$ CI 1.04 to $1.20, P=$ $0.003), 4$ cycles $(\mathrm{RR}=1.10,95 \% \mathrm{CI} 0.97$ to $1.24, P=$ 0.13)], PBC regimens [TP ( $R R=1.13,95 \%$ CI 1.06 to $1.21, P=0.0002), \mathrm{GP}(\mathrm{RR}=1.11,95 \% \mathrm{CI} 1.05$ to $1.18, P$ $=0.0004), \mathrm{NP}(\mathrm{RR}=1.10,95 \% \mathrm{CI} 1.02$ to $1.19, P=$ $0.003), \mathrm{EP}(\mathrm{RR}=1.22,95 \% \mathrm{CI} 1.02$ to $1.46, P=0.03)]$, or publication year (only including the studies published within 5 years) $(\mathrm{RR}=1.14,95 \%$ CI 1.07 to $1.22, P<$ 0.0001). 
Trial sequential analysis (TSA) indicated the required information size for a reliable and conclusive meta-analysis had been reached, and that CKI combined with $\mathrm{PBC}$ was significantly superior to $\mathrm{PBC}$ alone (Figure 10), suggesting that the findings of the meta-analysis are robust for the DCR. The meta-regression analysis showed that the DCR was not improved with an increased cycle number (from 2 to 4) of CKI ( $\log \mathrm{OR}=0.543, P=0.635$; Figure 11).

For the ORR, QOL, and severe toxicity, the sensitivity and subgroup analyses showed similar results. Due to the limited number of included studies, the subgroup and sensitivity analyses were not available for the outcomes 1-year survival rate.

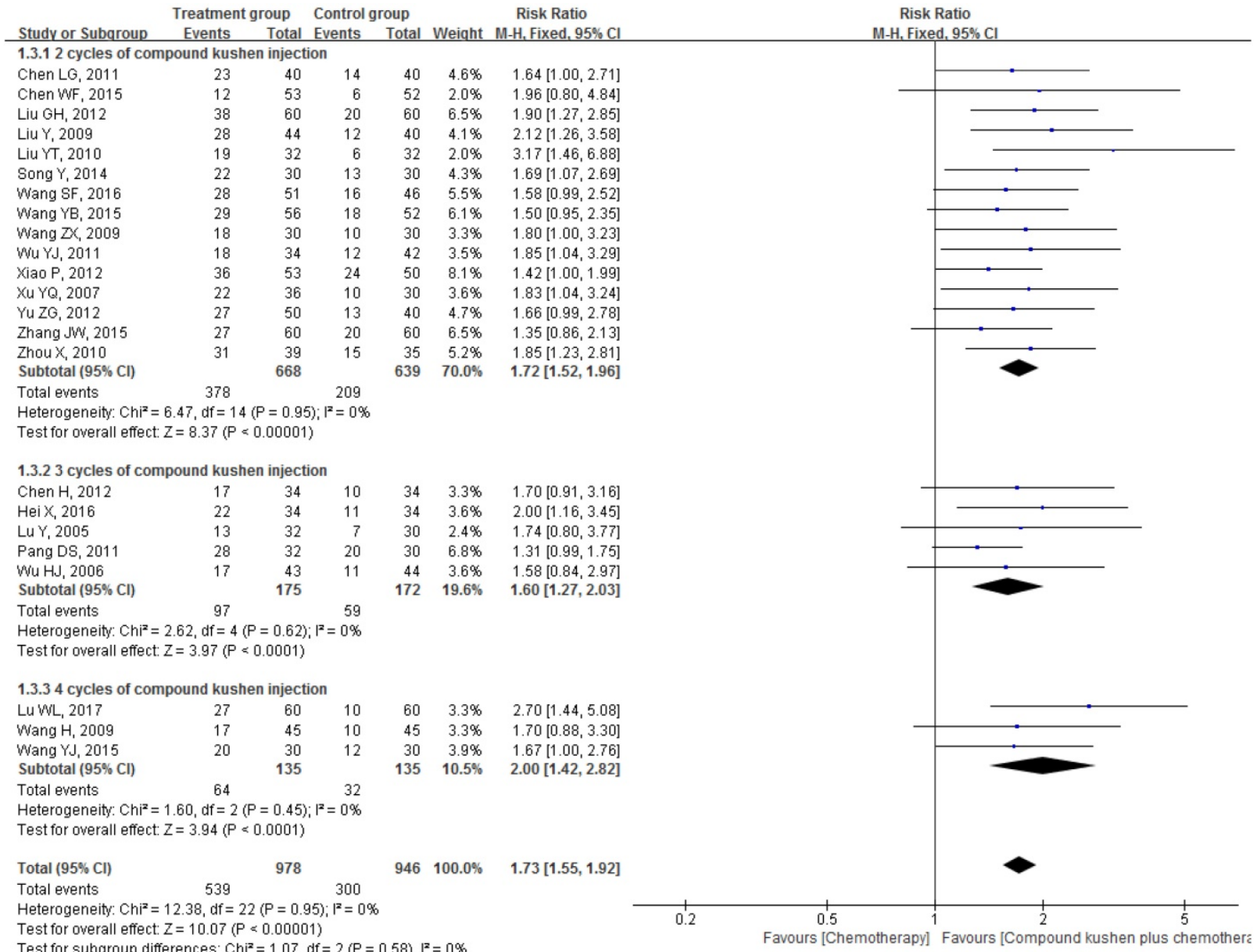

Figure 6. Forest plots showing a significant improvement in the $\mathrm{QOL}$ in the experimental group compared with that of the control group. Cl: Confidence Interval; CKI: Compound Kushen injection, PBC: platinum-based chemotherapy.

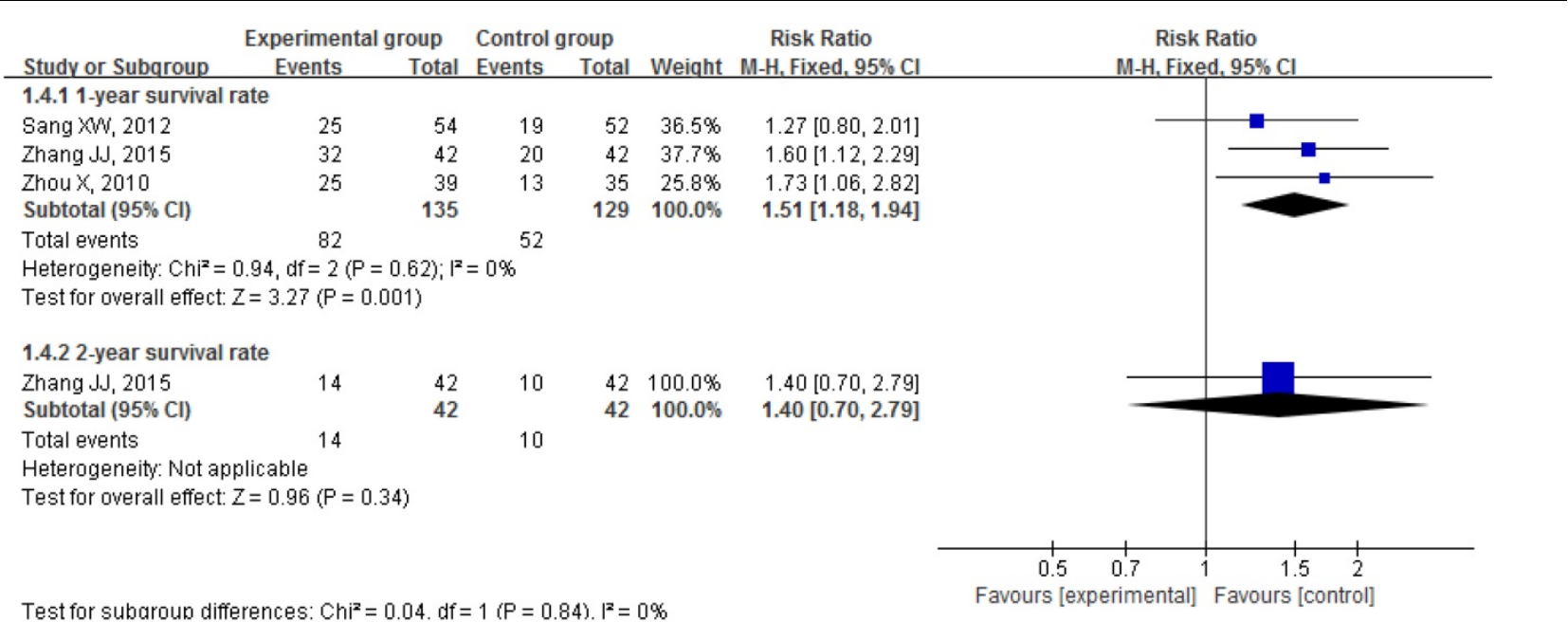

Figure 7. Forest plots showing a significant improvement in 1-year survival rate in the experimental group compared with that of the control group. Cl: Confidence Interval; CKI: Compound Kushen injection, PBC: platinum-based chemotherapy. 


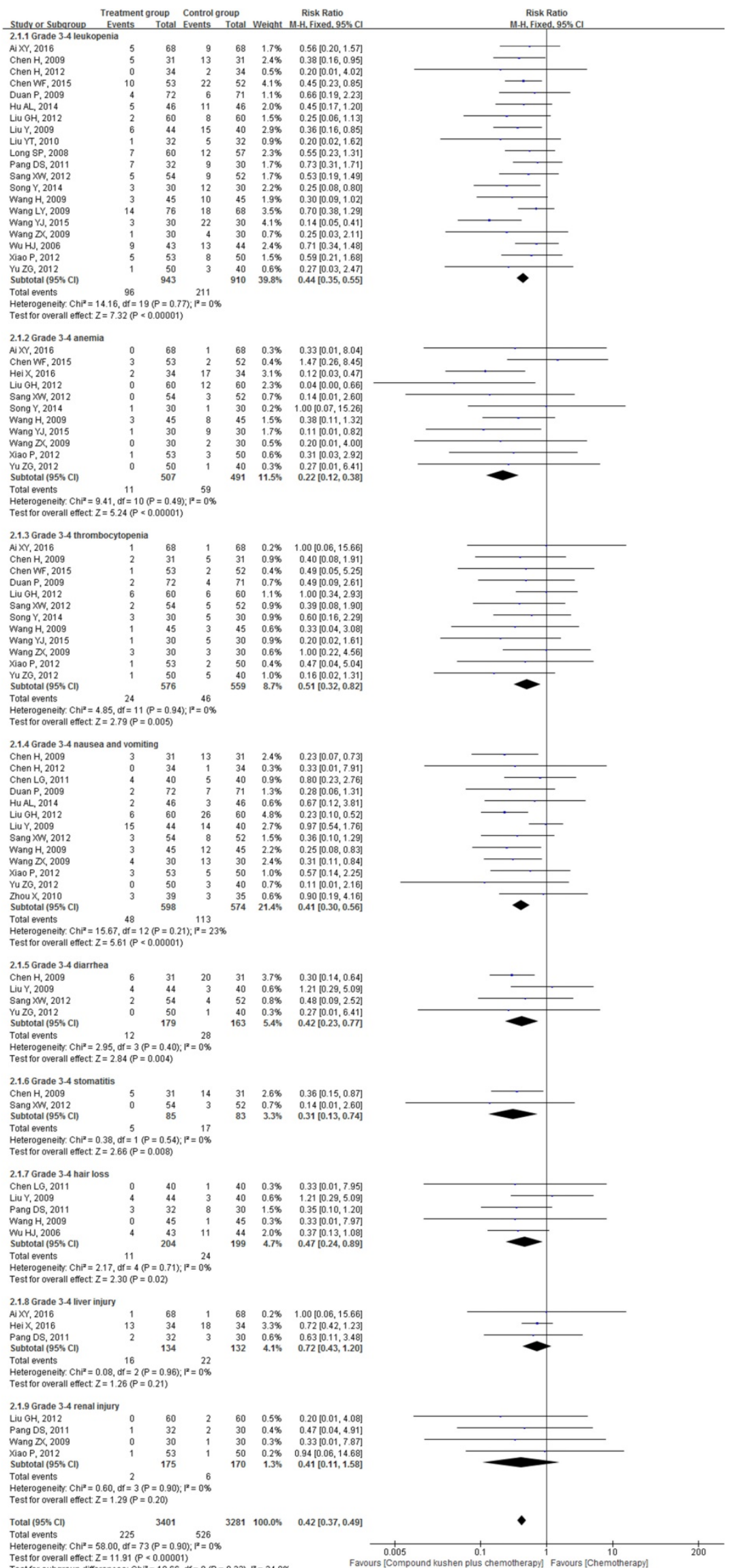

Figure 8. Forest plots showing a significant reduction of severe (grade 3 and 4 ) toxicities in the experimental group compared with those of the control group. Cl: Confidence Interval; CKI: Compound Kushen injection, PBC: platinum-based chemotherapy. 
a

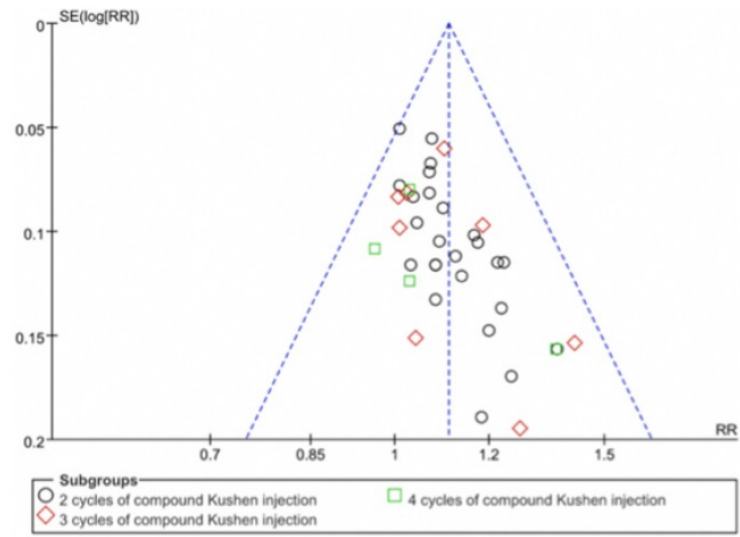

C

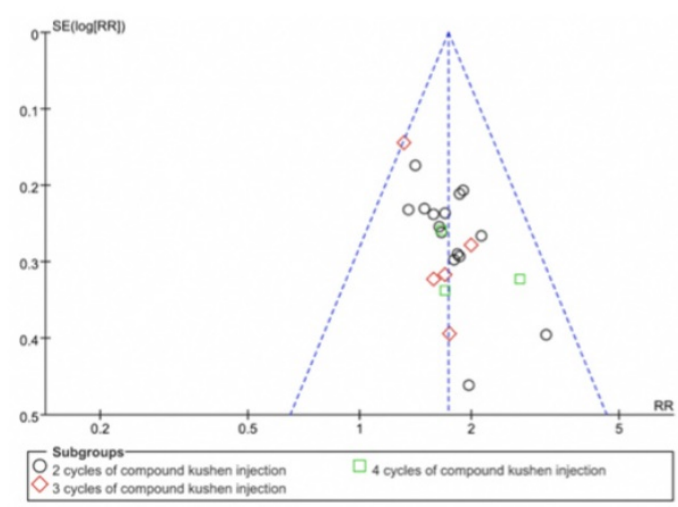

b

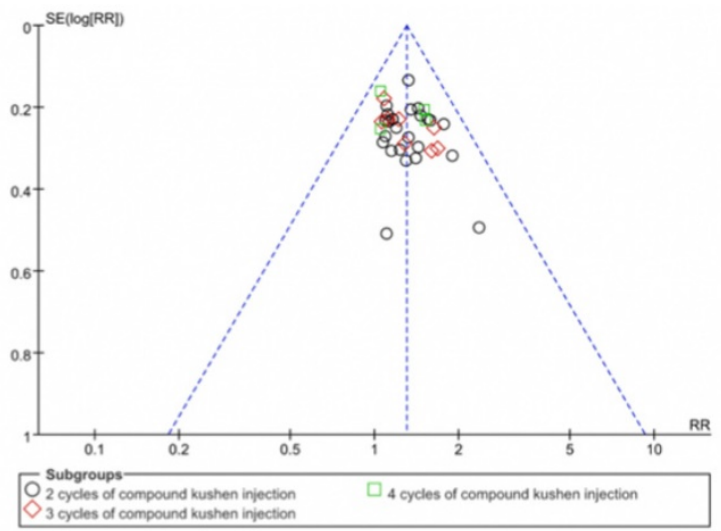

d

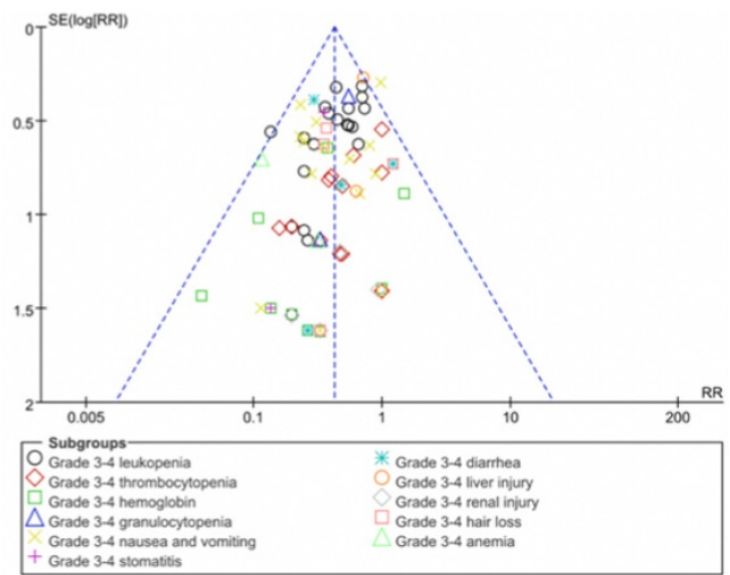

Figure 9. Funnel plots showing possible publication bias due to small-study effects. (A) Funnel plots of the DCR. (B) Funnel plots of the ORR. (C) Funnel plots of QOL. (D) Funnel plots of severe toxicities. RR: Relative Risk.

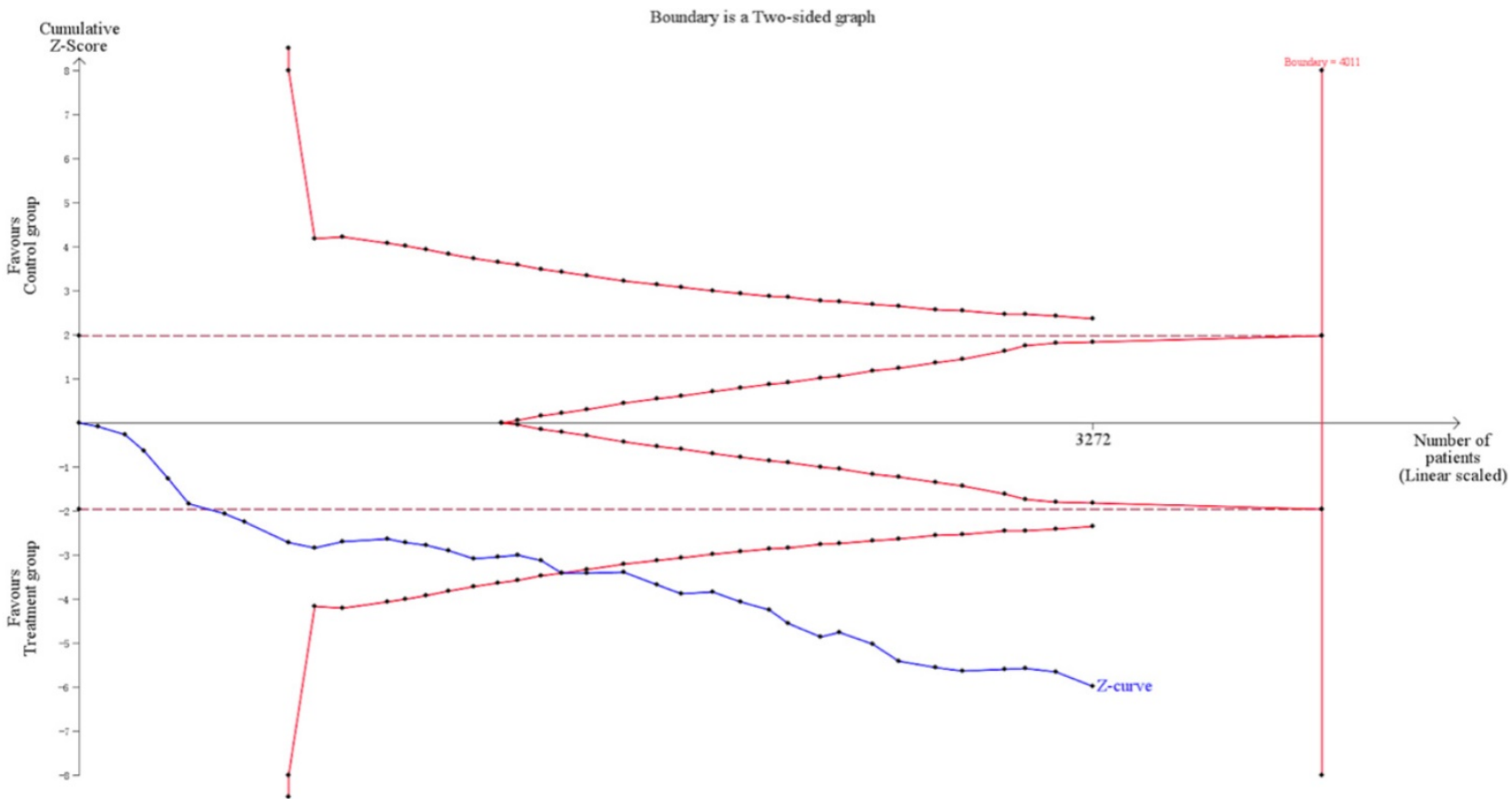

Figure 10. Trial sequential analysis of the DCR showing that the required information size had been reached and that Compound Kushen injection (CKI) plus PBC was significantly superior to the intervention in the control group. PBC, platinum-based chemotherapy. 
Regression of Log odds ratio on Cycle number

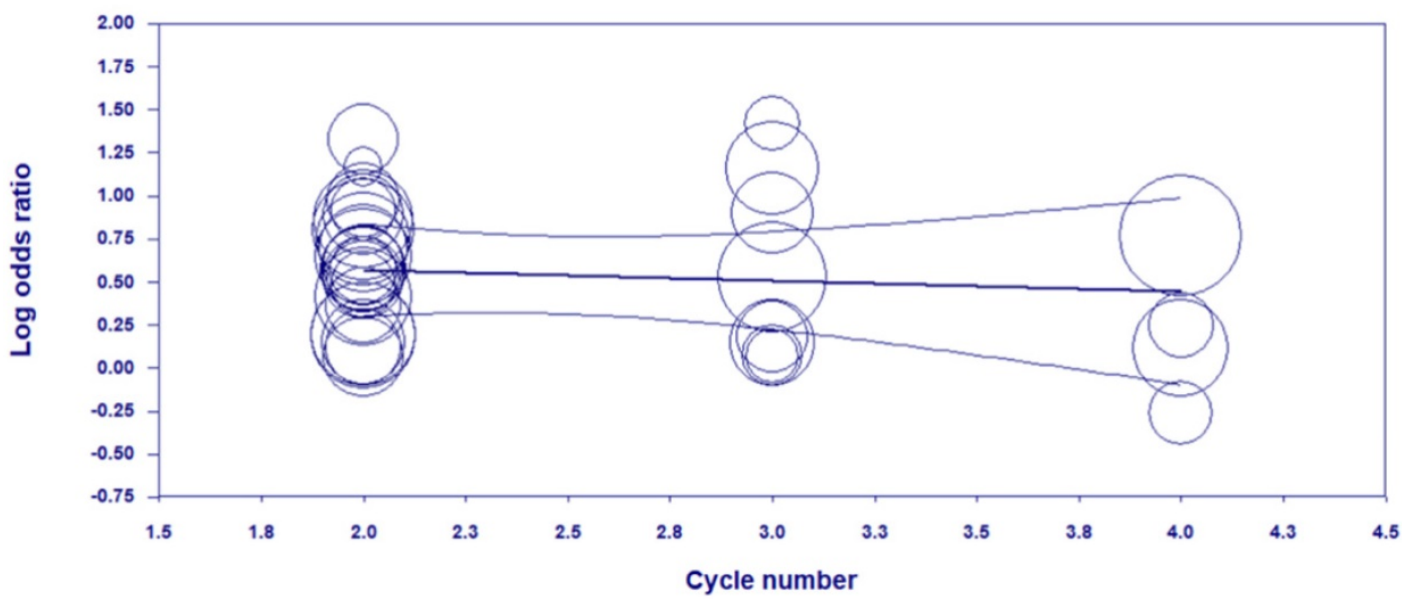

Figure 11. Meta-regression analysis showing that the DCR was not improved with an increased cycle number of Compound Kushen injection (CKI).

\section{Discussion}

As an important anti-tumor drug in China, CKI is broadly used for treating many kinds of cancer. The efficacy and safety of CKI combined with chemotherapy for many cancers, such as esophageal cancer, breast cancer, hepatocellular carcinoma, colon cancer, etc. have been systematically evaluated [24, 25, 77]. A previous meta-analysis evaluated the effects of CKI combined with radiotherapy for NSCLC, indicating that CKI plus radiotherapy significantly improved the clinical efficacy and reduced adverse events [78], however, the efficacy and safety of CKI plus PBC for advanced NSCLC patients have never been evaluated. The present study is the first systematic review and meta-analysis evaluating the effects of CKI plus PBC for advanced lung cancer patients. Our results showed that CKI as an adjunctive treatment to $\mathrm{PBC}$ can bring some crucial clinical benefits to the NSCLC patients at stage III/IV. This combined therapy could improve the tumor responses and QOL with a low risk of chemotherapy-induced toxicities in patients with advanced NSCLC. Our findings are consistent with the results of those meta-analyses evaluating the effects of CKI on other cancers [24, 25, 77, 79].

This meta-analysis included a total of 3,272 patients. Trial sequential analysis on DCR showed that CKI combined with PBC was significantly superior to $\mathrm{PBC}$ alone, and the findings of the meta-analysis are robust for the primary endpoint. The meta-regression analysis showed that the DCR was not improved with an increased cycle number (from 2 to 4 ), indicating 2 cycles of CKI might be an optimal treatment choice.

Both DCR and ORR are considered as important parameters for evaluating the short-term efficacy of antitumor therapy [14, 80-82]. DCR, defined as the proportion of patients with complete response, partial response or no change, is regarded as the best response categorization to predict OS and progression-free survival (PFS). [80, 81] Therefore, it was defined as the primary clinical endpoint in our study. The pooled results of our meta-analyses clearly demonstrated that CKI plus PBC could significantly improve DCR $(R R=1.11,95 \%$ CI 1.07 to 1.15$)$ and ORR ( $R R=1.30,95 \%$ CI 1.20 to 1.40$)$, suggesting that CKI increased the sensitivity of chemotherapy drugs and might also have synergic interactions with PBC. These are consistent with the results of previous meta-analyses evaluating the effects of CKI on the other cancers [24, 25, 77, 79], and the results of in vitro and in vivo experiments [24, 25]. Because the drug sensitivity is a major concern in chemotherapy for NSCLC $[83,84]$, the benefits observed with the CKI for these two short-term outcomes are of important clinical significance.

The follow-up duration of the included RCTs was relatively short (1-30 months) though some trials investigated the long-term efficacy of CKI and reported various survival outcomes. Because of the limited number of the included trials reporting the survival outcomes and/or unextractable data, meta-analysis was only available for 1-year survival rate, and the result of meta-analysis shows that, compared with PBC alone, CKI plus PBC significantly increased 1-year survival rate. This study could not comprehensively assess the long-term efficacy of CKI for advanced NSCLC. In the future, it is necessary to include and investigate more survival outcomes in the clinical trials to further assess the long-term efficacy of CKI $[84,85]$.

The adverse reactions caused by chemotherapy, such as gastrointestinal reactions and 
myelosuppression, are very common, which may lead to chemotherapy discontinuation, poor QOL, and the situation where harm outweighs the benefits of PBC in many patients treated by chemotherapy. The subgroup meta-analysis indicated that CKI plus PBC could significantly reduce adverse reactions, such as nausea and vomiting, leukopenia, thrombocytopenia, hemoglobin, stomatitis, diarrhea, hair loss, and anemia, etc. Our results demonstrated that CKI combined with $\mathrm{PBC}$ was associated with a significant improvement in QOL $(P<0.00001)$, suggesting that combined treatment increases the tolerance to chemotherapy.

This meta-analysis has some limitations: all included studies were conducted in China and published in Chinese, some of the included studies were of the potential risk of bias, and the follow-up durations of all included trials were relatively short, etc. Therefore, more rigorous trials with longer follow-up periods are warranted to further assess the efficacy and safety of this combination therapy. Recently, a multicenter randomized controlled trial has been initiated to assess the efficacy of CKI in combination with chemotherapy in the treatment of elderly patients with advanced NSCLC [86]. Such high-quality trials will help strengthen the evidence-base for this therapy [87].

\section{Conclusion}

From the available evidence, our meta-analysis indicates that Compound Kushen injection combined with platinum-based chemotherapy is more effective in improving clinical efficacy and alleviating the toxicity of chemotherapy than chemotherapy alone for the treatment of stage III/IV NSCLC. However, high-quality RCTs with survival outcomes are still needed to further confirm our findings.

\section{Abbreviations}

CI: Confidence Interval; CKI: Compound
Kushen injection; $\quad$ CMA: Comprehensive Meta-Analysis; CNKI: China National Knowledge Infrastructure; DC: Docetaxel plus carboplatin; DCR: Disease control rate; DP: Docetaxel plus cisplatin; EP: Etoposide plus cisplatin; GC: Gemcitabine plus carboplatin; GP: Gemcitabine plus cisplatin; ITT: Intention-to-treat; NP: Vinorelbine plus cisplatin; NSCLC: Non-small cell lung cancer; OR: Odds Ratio; ORR: Objective Response Rate; OS: Overall Survival; PBC: Platinum-based chemotherapy; PFS: Progression-free survival; PP: Pemetrexed plus cisplatin; QOL: Quality of life; RCT: Randomized controlled trial; RD: Risk Difference; RIS: Required information size; RM: Review Manager; RR: Risk Ratio; SMD: Standardized Mean Difference; T/C:
Treatment group/control group; TC: Paclitaxel plus carboplatin; TP: Paclitaxel plus cisplatin; TSA: Trial Sequential Analysis; WHO: World Health Organization; WMD: Weighted Mean Difference.

\section{Acknowledgements}

We would like to thank Ms. Judy Kodela, from The Acupuncture Office, PLLC, NY, USA, for proofreading our manuscript.

\section{Authors' Contributions}

Hongwei Chen, Xiaojun Yao, Ting Li: design, collecting data, statistical analysis, writing the article, final approval of the article.

Christopher Wai-Kei Lam: critical revision of the article, final approval of the article.

Huixia Zhang, Jue Wang: statistical analysis, final approval of the article.

Elaine Lai-Han Leung, Wei Zhang, Qibiao Wu: conception, design, critical revision of the article, final approval of the article, overall responsibility.

\section{Funding}

This work was funded by the Science and Technology Development Fund, Macau SAR (File No. 130/2017/A3, 0099/2018/A3, 0052/2018/A2 and 0096/2018/A3).

\section{Competing Interests}

The authors have declared that no competing interest exists.

\section{References}

1. Bray F, Ferlay J, Soerjomataram I, et al. Global Cancer Statistics 2018: GLOBOCAN Estimates of Incidence and Mortality Worldwide for 36 Cancers in 185 Countries. CA Cancer J Clin. 2018; 68:394-424. doi: 10.3322/caac.21492. [Epub ahead of print]

2. Siegel RL, Miller KD, Jemal A. Cancer statistics. CA Cancer J Clin. 2018; 68(1):7-30. doi: 10.3322/caac.21442. [Epub ahead of print]

3. Wu XP, Wu QB, Zhou XQ, et al. SphK1 functions downstream of IGF-1 to modulate IGF-1-induced EMT, migration and paclitaxel resistance of A549 cells: A preliminary in vitro study. J Cancer. 2019; 10:4264-4269. doi: 10.7150/jca.32646. eCollection 2019.

4. Cao M, Chen W. Epidemiology of lung cancer in China. Thorac Cancer. 2019 Jan; 10(1): 3-7. doi: 10.1111/1759-7714.12916. [Epub ahead of print]

5. Chen W, Zheng R, Zeng H, et al. Annual report on status of cancer in China, 2011. Chin J Cancer Res. 2015 Feb; 27(1):2-12. doi: 10.3978/j.issn.1000-9604.2015.01.06.

6. De Marinis F, Barberis M, Barbieri V, et al. Diagnosis and first-line treatment of non-small cell lung cancer in the era of novel immunotherapy: recommendations for clinical practice. Expert Rev Respir Med. 2019 Mar; 13(3): 217-228. doi: 10.1080/17476348.2019.1569517. [Epub ahead of print]

7. Gutierrez ME, Choi K, Lanman RB, et al. Genomic Profiling of Advanced Non-Small Cell Lung Cancer in Community Settings: Gaps and Opportunities. Clin Lung Cancer. 2017 Nov; 18(6): 651-659. doi: 10.1016/j.cllc.2017.04.004. [Epub ahead of print]

8. Hirsch FR, Zaric B, Rabea A, et al. Biomarker Testing for Personalized Therapy in Lung Cancer in Low- and Middle-Income Countries. Am Soc Clin Oncol Educ Book. 2017; 37: 403-408. doi: 10.14694/EDBK_175243.

9. Johnson M, Pennell NA, Borghaei H. "My Patient Was Diagnosed With Nontargetable Advanced Non-Small Cell Lung Cancer. What Now?" Diagnosis and Initial Treatment Options for Newly Diagnosed Patients With Advanced NSCLC. Am Soc Clin Oncol Educ Book. 2018 May 23; 38: 696-707. doi: 10.1200/EDBK_201231.

10. Kurtovic-Kozaric A, Vranic S, Kurtovic S, et al. Lack of Access to Targeted Cancer Treatment Modalities in the Developing World in the Era of Precision 
Medicine: Real-Life Lessons From Bosnia. J Glob Oncol. 2018 Sep; 4: 1-5. doi: 10.1200/JGO.2016.008698. [Epub ahead of print]

11. Schiller JH, Harrington D, Belani CP, et al. Comparison of four chemotherapy regimens for advanced non-small-cell lung cancer. N Engl J Med. 2002 Jan 10; 346(2): 92-8

12. de Castro J, Tagliaferri $\mathrm{P}$, de Lima V, et al. Systemic therapy treatment patterns in patients with advanced non-small cell lung cancer (NSCLC): PIvOTAL study. Eur J Cancer Care (Engl). 2017;26(6). doi: 10.1111/ecc.12734. [Epub ahead of print]

13. Leung EL, Wu QB. Concurrent use of herbal products with prescription drugs is a double-edged sword and evidence-based medicine contributes to reshaping the practice. Pharmacol Res. 2019 Mar;141:609-610. doi: 10.1016/j.phrs.2019.01.033. [Epub ahead of print]

14. Wang $X$, Liu $Z$, Sui $X$, et al. Elemene injection as adjunctive treatment to platinum-based chemotherapy in patients with stage III/IV non-small cell lung cancer: A meta-analysis following the PRISMA guidelines. Phytomedicine. 2018; 10; 59:152787. doi: 10.1016/j.phymed.2018.12.010. [Epub ahead of print]

15. Yang H, Xie Y, Ni J, et al. Association Rule Analysis for Validating Interrelationships of Combined Medication of Compound Kushen Injection in Treating Colon Carcinoma: A Hospital Information System-Based Real-World Study. Evid Based Complement Alternat Med. 2018 Aug 30;2018:4579801. doi: 10.1155/2018/4579801. eCollection 2018.

16. Gao L, Wang KX, Zhou YZ, et al. Uncovering the anticancer mechanism of Compound Kushen Injection against HCC by integrating quantitative analysis, network analysis and experimental validation. Sci Rep. 2018 Jan 12; 8(1): 624. doi: 10.1038/s41598-017-18325-7.

17. Wang W, You RL, Qin WJ, et al. Anti-tumor activities of active ingredients in Compound Kushen Injection. Acta Pharmacol Sin. 2015; 36: 676-9. doi: 10.1038/aps.2015.24. [Epub ahead of print]

18. Lu WL. Efficacy of Compound Matrine Injection in Combination with Gemcitabine and Cisplatin Chemotherapy in the Treatment of Patients with Advanced Non-small-cell lung Cancer. Liaoning J Tradit Chinese Medicine. 2017; 44:1214-1215.

19. Song Y. Clinical observation on treating advanced Non-small cell lung cancer with Compound Kushen injection plus chemotherapy. Clin J Chin Med. 2014; 6:4-6.

20. Cui I, Qu Z, Harata-Lee Y, et al. Cell cycle, energy metabolism and DNA repair pathways in cancer cells are suppressed by Compound Kushen Injection. BMC Cancer. 2019; 19:103. doi: 10.1186/s12885-018-5230-8.

21. Wang $\mathrm{H}, \mathrm{Hu} \mathrm{H}$, Rong $\mathrm{H}$, et al. Effects of compound Kushen injection on pathology and angiogenesis of tumor tissues. Oncol Lett. 2019; 17: 2278-2282. doi: 10.3892/ol.2018.9861

22. Luo $\mathrm{C}$, Zhu $\mathrm{Y}$, Jiang $\mathrm{T}$, et al. Matrine induced gastric cancer MKN45 cells apoptosis via increasing pro-apoptotic molecules of Bcl-2 family. Toxicology. 2007; 229: 245-252

23. Qu Z, Cui J, Harata-Lee Y, et al. Identification of candidate anti-cancer molecular mechanisms of Compound Kushen Injection using functional genomics. Oncotarget. 2016; 7:66003-66019. doi: 10.18632/oncotarget.11788

24. Ao M, Xiao X, Li Q. Efficacy and safety of compound Kushen injection combined with chemotherapy on postoperative Patients with breast cancer: A meta-analysis of randomized controlled trials. Medicine (Baltimore). 2019; e14024. doi: 10.1097/MD.0000000000014024

25. Ma X, Li RS, Wang J, et al. The Therapeutic Efficacy and Safety of Compound Kushen Injection Combined with Transarterial Chemoembolization in Unresectable Hepatocellular Carcinoma: An Update Systematic Review and Meta-Analysis. Front Pharmacol. 2016; 7: 70. doi: 10.3389/fphar.2016.00070. eCollection 2016

26. Zhao Z, Liao H, Ju Y. Effect of compound Kushen injection on T-cell subgroups and natural killer cells in patients with locally advanced non-small-cell lung cancer treated with concomitant radiochemotherapy. J Tradit Chin Med. 2016; 36: 14-8.

27. Dong J, Lou T. Treatment of 40 Cases of Advanced Non-small Cell Lung Cancer with Compound Kushen Injection and PD. Zhejiang J Integrated Tradit Chin and Western Med. 2012: 22: 778-780.

28. Lu Y, Zhang XY, Wang C. Clinical observation of compound Kushen injection combined with chemotherapy in the treatment of non-small cell lung cancer. Ningxia Med J. 2005; 27: 252-253.

29. Pang DS, Xu Y, Wang XY, et al. Clinical Effects of TP Project Combined with Compound Matrine Injections on Advanced Non-Small-Cell Lung Cancer. China J General Practice. 2011; 1:50-164

30. Wang H, Tao L. Compound Kushen Injection Combined with Chemotherapy for Non-small Cell Lung Cancer Clinical Observation. Chin Med Innovations. 2009; 6:14-16

31. Wu HJ, Zou HZ, Liu T. Clinical Observation of Late Non-small-cell Lung Cancer Treated by Chemotherapy Combined with Yanshu Injection. Evaluation and Analysis of Drug-Use in Hospitals of China. 2006; 6: 43-45.

32. Xiao P, Meng JQ, Meng XR, et al. Efficacy and Toxicity of Compound Matrine Injection Combined with Gemcitabine Plus Cisplatin for Advanced Non-Small-Cell Lung Cancer: Analysis of 56 Cases. Evaluation and Analysis of Drug-Use in Hospitals of China. 2012; 12:155-157.

33. Moher D, Liberati A, Tetzlaff J, et al. Preferred reporting items for systematic reviews and meta-analyses: the PRISMA statement. PLoS Med. $2009 \mathrm{Jul}$ 21;6(7):e1000097. doi: 10.1371/journal.pmed.1000097. [Epub ahead of print]
34. Fleming ID, Cooper JS, Henson DE, et al. AJCC cancer staging manual. 5th ed. Philadelphia (PA): Lippincott-Raven. 1997.

35. Greene FL, Page DL, Fleming ID, et al. AJCC Cancer Staging Manual. 6th ed. New York, NY: Springer-Verlag. 2002.

36. Sobin LH, Fleming ID. International Union Against Cancer (UICC). TNM classification of malignant tumors. 5th ed. New York: John Wiley \& Sons. 1997.

37. Yates JW, Chalmer B, McKegney FP. Evaluation of patients with advanced cancer using the Karnofsky performance status. Cancer. 1980; 45: 2220-2224.

38. Miller AB, Hoogstraten B, Staquet $M$, et al. Reporting results of cancer treatment. Cancer. 1981; 47:207-214.

39. World Health Organization. World Health Organization Handbook for Reporting Results of Cancer Treatment. WHO Offset Publication no. 48, Geneva, Switzerland. 1979.

40. Higgins J, Green S. Cochrane Handbook for Systematic Reviews of Interventions Version 5.1.0. (2011). http://www.cochrane-handbook.org. (Accessed 18.09.08). 2011.

41. Riley RD, Higgins JP, Deeks JJ. Interpretation of random effects meta-analyses. BMJ. 2011; 10; 342: d549. doi: 10.1136/bmj.d549.

42. Wu QB, Li GC, Lei WI, et al. The efficacy and safety of tiotropium in Chinese patients with stable chronic obstructive pulmonary disease: a meta-analysis. Respirology. 2009; 14:666-74. doi: 10.1111/j.1440-1843.2009.01525.x.

43. Zhou Z, Shen WX, Yu LL, et al. 2016. A Chinese patent medicine, Shexiang Baoxin Pill, for Non-ST-elevation acute coronary syndromes: A systematic review. J Ethnopharmacol. 2016; 194:1130-1139. doi: 10.1016/j.jep.2016.11.024.

44. Begg CB, Mazumdar M. Operating characteristics of a rank correlation test for publication bias. Biometrics. 1994; 50: 1088-1101.

45. Egger M, Davey Smith G, Schneider M, et al. Bias in meta-analysis detected by a simple, graphical test. British Medical Journal. 1997; 315:629-634.

46. Furlan AD, Pennick V, Bombardier C, et al. 2009 updated method guidelines for systematic reviews in the Cochrane Back Review Group. Spine (Phila Pa 1976) 2009 Aug 15; 34(18):1929-41. doi: 10.1097/BRS.0b013e3181b1c99f.

47. Thorlund K, Engstrøm J, Wetterslev J, et al. User manual for trial sequential analysis (TSA). Copenhagen Trial Unit, Centre for Clinical Intervention Research, Copenhagen, Denmark. Available from www.ctu.dk/tsa. (Accessed 18.09.30). 2011; p. 1-115

48. Ai XY, et al. Clinical Effect of Compound Kushen Injection Combined with Chemotherapy in Advanced Non-Small Cell Lung Cancer. Liaoning J Tradit Chin Med. 2016; 8, 1690-1691.

49. Chen H, Ren ZC, Li R,et al. Treatment of 31 cases of advanced non-small cell lung cancer with integrated traditional Chinese and Western medicine. Yunnan J Traditi Chin Med and Materia Med. 2009; 30:31.

50. Chen LG, Zhang YM, Zhu TN. Clinical Effect of Compound Kushen Injection Combined with Chemotherapy in Lung Cancer. The Asia-pacific Tradit Med. 2011; 7: 66-67.

51. Chen $\mathrm{H}$, Zhao $\mathrm{L}$, Luo $\mathrm{T}$, et al. Clinical Effects of Compound Matrine Injections Combined with TP Chemotherapy on Non-Small-Cell Lung Cancer. Chin Med Modern Distance Education of China. 2012; 10: 148-149.

52. Chen WF, Zhu L. Treatment of 53 cases of non-small cell lung cancer with compound Kushen injection combined with chemotherapy. Liaoning J Tradit Chin Med. 2015; 42:126-127

53. Duan P, Xiao MW, Xiao W, et al. Efficacy of compound Kushen injection in combination with chemotherapy in patients with advanced non-small cell lung cancer. Chin J New Drugs. 2009; 18.

54. Hei X. Clinical efficacy of compound Kushen injection and chemotherapy in the treatment of advanced non-small cell carcinoma. World Latest Med Information. 2016; 16:123-124.

55. Hu AL. Clinical Observation of Compound Kushen Combined with Chemotherapy in Patients with Advanced Lung Cancer. Public Med Forum Magazine. 2014; 18:1270-1271.

56. Huang ZF, Liu JB, Li HZ, et al. Treating 30 cases of NSCLC in stage III and IV with Compound Kushen injection plus Chemotherapy. China Association of Chin Med. 2007; 3:319-321.

57. Li CJ, Liu ZP, Jin HY, et al. Clinical observation of 40 cases of advanced non-small cell lung cancer. Contemporary Med. 2011; 17: 108.

58. Liu Y, Wei Z, Meng LZ, et al. Clinical observation of Yanshu injection combined with chemotherapy in the treatment of advanced non-small cell lung cancer. Shanxi J Tradit Chin Med. 2009; 25:24-25.

59. Liu YT, Zhang HY, Liu YL. Treatment of 30 Cases of Advanced Non-small Cell Lung Cancer with Compound Kushen Injection Combined with Chemotherapy. J Clin Pulmonary Med. 2010; 15:727-728.

60. Liu GH, Li T. Efficacy of compound matrine injection in combination with gemcitabine and cisplatin chemotherapy in the treatment of patients with advanced non-small-cell lung cancer. Chin J New Drugs. 2012; 21:658-665.

61. Long SP, Zeng JQ. Therapeutic effect of compound Kushen injection combined with TP regimen in the treatment of non-small cell lung cancer. Jiangxi Med J. 2008; 919-920

62. Sang XW. Addition of Compound Kushen Injection to Vinorelbine Plus Cisplatin for Patients with Non-small Cell Lung Cancer: Clinical Observation. Evaluation and Analysis of Drug-Use in Hospitals of China. 2012; 12: 645-648.

63. Su WZ, Li XN. Effect of Yanshu Injection Combined with NP Regimen on Advanced Non-small Cell Lung Cancer. Med Recapitulate. 2012; 13:1891-1892.

64. Wang ZX, Li J, Lu BB, et al. Efficacy and Detoxicating Effect of Compound Matrine Injection in Combination with Gemcitabine and Cisplatin Chemotherapy in the Treatment of Patients with Advanced Non-small-cell 
Lung Cancer. Evaluation and Analysis of Drug-Use in Hospitals of China. 2009; 9: 932-933.

65. Wang LY, Pan YP, Liu WH. Clinical Analyses of Chemotherapy Combined with Compound Sophora Injection in the Treatment of Advanced Non-small-cell Lung Cancer. Liaoning J Tradit Chin Med. 2009; 36: 2098-2100.

66. Wang YJ. Clinical study of compound Kushen injection combined with chemotherapy in the treatment of advanced non-small cell lung cancer. Tianjin Pharm. 2015; 27:35-36.

67. Wang YB. Clinical Study of Compound Kushen Injection Combined with Chemotherapy on Advanced Non-small Cell Lung Cancer. China J Chin Med. 2015; 30:1710-1711.

68. Wang HJ, Li YH, Wang JH, et al. Effect of matrine injection on the life quality of patients with advanced non-small cell lung cancer. Clin Focus. 2012; 27:9-12.

69. Wang SF, Hou CY, Ke YG. Clinical effect of Fufang Kushen injection combined with chemotherapy on bone metastases in non-small cell lung cancer pain. J Modern Oncology. 2016; 24: 49-52.

70. Wu YJ, Cui R, Yang YX, et al. Efficacy of Compound Kushen Injection combined with TP/GP regimen in the treatment of advanced non-small cell lung cancer. Shandong Med J. 2011; 51:88-89.

71. Zhang JW, Duan DM, Ren ZH. Effect of Kushen Injection Combined with GP Regimen in Patients with Non-small Cell Lung Cancer. Chin J Experimental Tradit Med Formulae. 2015; 21:184-187.

72. Zhang JJ, Yang JL, Yuan XM. Clinical observation of compound Kushen injection in the treatment of advanced non-small cell lung cancer. J New Chin Med. 2015; 47: 190-192.

73. $\mathrm{Xu} \mathrm{YQ}$, Ding R, Luo Y. Yanshu Injection in Combination with TP Chemotherapy for Advanced Non -Small Cell Lung Cancer: Evaluation of Clinical Efficacy. Evaluation and Analysis of Drug-Use in Hospitals of China. 2007; 25-26.

74. Yu ZG, Wang BC. Efficacy of Large Dose of Compound Matrine Injection Combined Chemotherapy for Non-Small-Cell Lung Cancer. Evaluation and Analysis of Drug-Use in Hospitals of China. 2012; 12:149-152.

75. Zhao K, Yang JQ, Wang JH, et al. Influence of Compound Matrine Injection Combined with GP Regimen on Therapeutic Efficacy and the Quality of Life in Patients with Advanced Non-small Cell Lung Cancer. Practical Prev Med. 2012; 19:1853-1854.

76. Zhou X, Liu CL. Clinical observation of 41 cases of advanced non-small cell lung cancer treated with GP regimen combined with Yanshu injection. Chin J Clin Oncology. 2010; 37:531-532.

77. Yu L, Zhou Y, Yang Y, et al. Efficacy and Safety of Compound Kushen Injection on Patients with Advanced Colon Cancer: A Meta-Analysis of Randomized Controlled Trials. Evid Based Complement Alternat Med. 2017: 7102514. doi: 10.1155/2017/7102514.

78. Wang S, Lian X, Sun M, et al. Efficacy of compound Kushen injection plus radiotherapy on nonsmall-cell lung cancer: A systematic review and meta-analysis. J Cancer Res Ther. 2016; 12:1298-1306. doi: 10.4103/0973-1482.199538.

79. Zhang D, Ni M, Wu J, et al. The Optimal Chinese Herbal Injections for Use with Radiotherapy to Treat Esophageal Cancer: A Systematic Review and Bayesian Network Meta-Analysis. Front Pharmacol. 2019 Jan 4; 9:1470. doi: 10.3389/fphar.2018.01470. eCollection 2018.

80. Claret L, Gupta M, Han K, et al. Prediction of overall survival or progression free survival by disease control rate at week 8 is independent of ethnicity: Western versus Chinese patients with first-line non-small cell lung cancer treated with chemotherapy with or without bevacizumab. J Clin Pharmacol. 2014; 54:253-257. doi: 10.1002/jcph.191. Epub 2013 Oct 22.

81. Paesmans M, Sculier JP, Libert P, et al. Response to chemotherapy has predictive value for further survival of patients with advanced non-small cell lung cancer: 10 years experience of the European Lung Cancer Working Party. Eur J Cancer. 1997; 33:2326-32.

82. Wang J, Li G, Yu L, et al. Aidi injection plus platinum-based chemotherapy for stage IIIB/IV non-small cell lung cancer: A meta-analysis of 42 RCTs following the PRISMA guidelines. J Ethnopharmacol. 2018; 221:137-150. doi: 10.1016/j.jep.2018.04.013. Epub 2018 Apr 12.

83. Sosa Iglesias V, Giuranno L, Dubois LJ, et al. Drug Resistance in Non-Small Cell Lung Cancer: A Potential for NOTCH Targeting? Front Oncol. 2018; 8:267. doi: 10.3389 /fonc. 2018.00267

84. Broglio KR, Berry DA. Detecting an overall survival benefit that is derived from progression-free survival. J Natl Cancer Inst. 2009; 101:1642-1649. doi: 10.1093/jnci/djp369. Epub 2009 Nov 9.

85. Chen HW, Yao XJ, Liu ZT, Li T, Xu C, Wang J, Sui XB, Leung ELH, Wu QB. Efficacy and safety of Shenqi Fuzheng injection combined with platinumbased chemotherapy for stage III/IV non-small cell lung cancer. Medicine. 2019; e17350. doi: 10.1097/MD.0000000000017350

86. Wang XQ, Liu J, Lin HS, et al. A multicenter randomized controlled open-label trial to assess the efficacy of compound kushen injection in combination with single-agent chemotherapy in treatment of elderly patients with advanced non-small cell lung cancer: study protocol for a randomized controlled trial. Trials. 2016 Mar 8; 17(1): 124. doi: 10.1186/s13063-016-1231-6.

87. Wu $\mathrm{Q}$, Leung EL. The evidence strength of a meta-analysis of aspirin for primary prevention of cancer. J Cancer Res Clin Oncol. 2019 Oct 31. doi: 10.1007/s00432-019-03069-w. [Epub ahead of print]. 